\title{
GEOPHYSICS $^{\odot}$
}

Target-oriented time-lapse waveform inversion using deep learning assisted regularization

\begin{tabular}{|r|l|}
\hline Journal: & Geophysics \\
\hline Manuscript ID & GEO-2020-0383.R2 \\
\hline Manuscript Type: & Technical Paper \\
\hline Keywords: & time-lapse, full-waveform inversion, datuming, machine learning \\
\hline Manuscript Focus Area: & Seismic Inversion \\
\hline
\end{tabular}

SCHOLARONE $^{\text {m }}$
Manuscripts 


\title{
Target-oriented time-lapse waveform inversion using deep learning assisted regularization
}

\author{
Yuanyuan $\mathrm{Li}^{1 *}$, Tariq Alkhalifah ${ }^{1}$ and Qiang $\mathrm{Guo}^{2}$ \\ Running head: Target-oriented time-lapse waveform inversion \\ 1 King Abdullah University of Science and Technology, Physical Science and Engineering \\ Division, 4700, KAUST, Thuwal, 23955-6900, Kingdom of Saudi Arabia. \\ E-mail: yuanyuan.li@kaust.edu.sa; tariq.alkhalifah@kaust.edu.sa. \\ 2 Formerly in King Abdullah University of Science and Technology, KAUST; Currently in \\ Shearwater Geoservices. E-mail: qiang.guo@kaust.edu.sa. \\ *Corresponding author: yuanyuan.li@kaust.edu.sa.
}




\begin{abstract}
Detection of the property changes in the reservoir during injection and production is important. However, the detection process is very challenging using surface seismic surveys because these property changes often induce subtle changes in the seismic signals. The quantitative evaluation of the subsurface property obtained by full waveform inversion (FWI) allows for better monitoring of these time-lapse changes. However, high-resolution inversion is usually accompanied with a large computational cost. Besides, the resolution of inversion is limited by the bandwidth and aperture of time-lapse seismic data. We apply a target-oriented strategy through seismic redatuming to reduce the computational cost by focusing our high-resolution delineation on a relatively small zone of interest. The redatuming technique generates time-lapse virtual data for the target-oriented inversion. Considering the injection and production wells are often present in the target zone, we can incorporate the well velocity information to the time-lapse inversion by using regularization to complement the resolution and illumination at the reservoir. We use a deep neural network $(\mathrm{DNN})$ to learn the statistical relationship between the inverted model and the facies interpreted from well logs. The trained network is employed to map the property changes extracted from the wells to the target inversion domain. We then perform another time-lapse inversion, in which we fit the predicted data difference to the redatumed one from observation, as well as fit the model to the predicted velocity changes. The numerical results demonstrate that the proposed method is capable of inverting for the time-lapse property changes effectively in the target zone by incorporating the learned model information from well logs.
\end{abstract}




\section{INTRODUCTION}

Time-lapse seismic data have been widely used to monitor the fluid injection and the hydrocarbon extraction in the oilfield development and evaluate the storage of $\mathrm{CO}_{2}$ in carbon capture and storage (CCS) (Lumley, 2001; Arts et al., 2003; Chadwick et al., 2010; Urosevic et al., 2010). Time-lapse full waveform inversion (TLFWI) provides a quantitative estimation of the subsurface property changes induced by the aforementioned operations by fitting the time-lapse seismic data directly (Hicks et al., 2016; Maharramov et al., 2016; Kazei and Alkhalifah, 2018). High-resolution delineation of the property changes is a major goal of time-lapse seismic inversion. However, there are two primary issues that hinder the time-lapse inversion in practice. First, high-resolution full waveform inversion (FWI) is computationally intensive, because fine discretization is required to stabilize the simulation of high-frequency wavefields (Alford et al., 1974; Routh et al., 2017). Its corresponding time-lapse applications require even more computational cost, because we often need two FWI runs on the monitor and baseline data. Second, due to the limitation we encounter with surface seismic data in terms of signal-to-noise ratio (SNR), frequency band, aperture and coverage, seismic data nearly always admit incomplete subsurface information with limited resolution (Sirgue and Pratt, 2004; Alkhalifah, 2016; Li et al., 2018). TLFWI is a severely ill-posed inverse problem due to the incomplete measured data and the large search space (Tarantola, 1984; Zhang and Huang, 2013). In this work, we improve the performance of TLFWI by addressing these two issues.

The target-oriented inversion strategy is capable of reducing the computational cost by inverting for a relatively small target zone of the subsurface (van Manen et al., 2007; Ayeni and Biondi, 2010; Borisov and Singh, 2015; Yuan et al., 2017; Biondi et al., 2018; 
da Costa et al., 2019; Guo and Alkhalifah, 2020; Li et al., 2020b). The target-oriented strategy can be applied to time-lapse seismic inversion because the monitored property changes usually occur in the reservoir of interest (Li et al., 2020b, 2020c). Redatuming techniques generate a virtual dataset for the target-oriented inversion by projecting sources and receivers to a desired datum level just above the target area (Wapenaar and Fokkema, 2006; Yang et al., 2012; Guo and Alkhalifah, 2020). In addition, the wavefield distortion from the complex overburden, above the datum level, is also suppressed by waveform redatuming, and thus, has less of an effect on the target inversion (Zhao et al., 2020). The redatuming is expected to improve time-lapse repeatability by suppressing 4D unrepeatable noise such as that induced by near-surface diurnal and seasonal weather cycles as well as small changes in acquisition geometry and shot coupling (Bakulin and Calvert, 2006). Li et al. (2020b) investigate the impacts of the overburden model and 4D noise on the process of redatuming and target-oriented time-lapse inversion.

In TLFWI, the time-lapse property changes are given by the difference of the monitor and baseline FWI resulting models. The two FWI runs for monitor and baseline can be performed in parallel or in a coupled fashion (Denli and Huang, 2009; Zheng et al., 2011; Routh et al., 2012; Asnaashari et al., 2014; Hicks et al., 2016), often depending on their assumptions about the repeatability of the acquisition survey. Double-difference waveform inversion (DDWI; Denli and Huang, 2009) renders the estimated property changes less contaminated by the imperfect inverted baseline model. However, 4D unrepeatable noise deteriorates its performance (Yang et al., 2015).

Prior information needs to be incorporated into the inversion through regularization to steer inversion towards geologically realistic models. Total variation (TV) regularization 
has been used to preserve sharp boundaries in the inversion result (Lin and Huang, 2015; Esser et al., 2018; Kalita et al., 2019). Zhang and Huang (2013) incorporate the location of a monitoring region as prior information into double-difference waveform inversion to improve the inversion quality. Considering the injection and production wells are often present in the target zone, well velocity information, containing fine-scale model information, can be incorporated into the inversion to complement the resolution and illumination at the target monitoring zone (Asnaashari et al., 2013; Zhang et al., 2018; Singh et al., 2018; Aragao and Sava, 2020). The model information in the sparsely sampled wells should be projected to the inversion region to provide a prior model for regularization of the inversion.

Deep learning (DL; LeCun et al., 2015) can efficiently learn a statistical relationship between the input and output features in a data-driven manner (Zhang and Alkhalifah, 2019; Li et al., 2020a; Song et al., 2021). Zhang and Alkhalifah (2019) employ deep neural networks (DNNs) to build the proper statistical connection that converts seismic estimates to facies interpreted from well logs, and derived a prior model that incorporates the well information. The prior model helps the subsequent elastic full waveform inversion converge to a more accurate inversion result. Facies are defined as groups of seismic properties and conformity layers that share a particular relationship with geologic and lithologic properties (Kemper and Gunning, 2014). To retain the high-resolution well velocity in the prior model, Li et al. (2020a) extract the statistical distribution information, defined in a Gaussian window, from the wells and mapped the interpreted facies featured by the distribution information (mean and variance) to the inversion region to recover a 
prior model. The high-resolution prior information is then added to elastic full waveform inversion scheme as a regularization term.

In our proposed method, we adopt waveform redatuming to retrieve the targetoriented data for the time-lapse full waveform inversion focused on the target zone. Thus, we reduce the computational burden of the time-lapse full waveform inversion by inverting for a relatively small zone of interest. We also incorporate well velocity information to constrain the TLFWI scheme by using a DL-assisted regularization technique to improve the inversion accuracy and resolution. The required prior model is predicted from a trained DNN that learns the statistical relationship between the time-lapse seismic estimation and the facies interpreted from well logs. Numerical tests on the time-lapse Marmousi and SEAM models are used to demonstrate the performance of the proposed method.

\section{THEORY}

In this section, we first briefly review the redatuming scheme in the time-space domain, which provides the virtual dataset at the predesigned datum level for the targetoriented full waveform inversion. Then, we introduce regularized time-lapse full waveform inversion method, that incorporates well velocity information into inversion using deep learning.

\section{Waveform redatuming}

A typical configuration for waveform redatuming is shown in Figure 1. The sources and receivers for a surface acquisition system are located near the Earth's surface. The redatuming operation aims to redatum the surface seismic data from the Earth's surface to a 
desired datum level just above the target area by removing the effects of the overburden on the wavefields. The overburden denotes the zone above the datum level. It is an inverse problem that predicts the virtual dataset (Green's function) at the datum level using the observed seismic data and a reasonable estimate of the overburden model. To solve this inverse problem, the corresponding modeling operator needs to be constructed. Based on the convolution-type representation of Green's functions (Wapenaar and van der Neut, 2010), the upgoing wavefield $v$ at a virtual receiver $\mathbf{x}_{v r}$ excited by the source at $\mathbf{x}_{s}$ can be written as a convolution-type representation (Wapenaar and van der Neut, 2010):

$$
v\left(\mathbf{x}_{v r}, \mathbf{x}_{s}, t\right)=\int_{S_{D}} g_{D}\left(\mathbf{x}_{v r}, \mathbf{x}_{v s}, t\right) * u\left(\mathbf{x}_{v s}, \mathbf{x}_{s}, t\right) d \mathbf{x}_{v s},
$$

where, * refers to a temporal convolution operator, $S_{D}$ denotes the surface at datum level, $\mathbf{x}_{v s}$ represents the virtual source location, $u$ represents the downgoing wavefield from surface to datum and $\mathrm{g}_{D}$ indicates the unknown Green's function at the datum level describing scattering from the underlying model. The downgoing wavefield $(u)$ and the upgoing wavefield $\left(u_{D}\right)$ from the datum are obtained by solving the following wave equations:

$$
\begin{aligned}
& \mathbf{F} u\left(\mathbf{x}, \mathbf{x}_{s}, t\right)=f\left(\mathbf{x}, \mathbf{x}_{s}, t\right), \\
& \mathbf{F} u_{D}\left(\mathbf{x}, \mathbf{x}_{s}, t\right)=\int_{S_{D}} \delta\left(\mathbf{x}-\mathbf{x}_{v r}\right) v\left(\mathbf{x}_{v r}, \mathbf{x}_{s}, t\right) d \mathbf{x}_{v r},
\end{aligned}
$$

where $\mathbf{F}$ is the acoustic wave modeling operator $\mathbf{F}=\left(\frac{1}{c^{2}} \frac{\partial^{2}}{\partial t^{2}}-\nabla^{2}\right), c$ is the velocity, $f$ denotes the source function and $\delta\left(\mathbf{x}-\mathbf{x}_{v r}\right)$ is a Dirac delta function. Substituting equation 1 into 3, the upgoing wavefield $\left(u_{D}\right)$ is written as: 


$$
\mathbf{F} u_{D}\left(\mathbf{x}, \mathbf{x}_{s}, t\right)=\int_{S_{D}} \delta\left(\mathbf{x}-\mathbf{x}_{v r}\right) \int_{S_{D}} g_{D}\left(\mathbf{x}_{v r}, \mathbf{x}_{v s}, t\right) * u\left(\mathbf{x}_{v s}, \mathbf{x}_{s}, t\right) d \mathbf{x}_{v s} d \mathbf{x}_{v r}
$$

The wavefield that is related to the response of the overburden provides a pilot trace at the virtual source location. Then, the modeling operator can be given as (Guo and Alkhalifah, 2019):

$$
\mathbf{F} u_{D}\left(\mathbf{x}, \mathbf{x}_{s}, t\right)=\iint u\left(\mathbf{x}_{v s}-\mathbf{h}, \mathbf{x}_{s}, t\right) * g_{D}\left(\mathbf{x}_{v s}-\mathbf{h}, \mathbf{x}_{v s}, t\right) \delta\left(\mathbf{x}-\mathbf{x}_{v s}+\mathbf{h}\right) d \mathbf{h} d \mathbf{x}_{v s}
$$

where $\mathbf{h}$ is the subsurface offset vector pointing from the virtual source. The simulated upgoing wavefield $\left(u_{D}\right)$ is generated from the secondary source, located at the datum level, given by the convolution of the downgoing wavefield $(u)$ with the Green's function $\left(\mathrm{g}_{D}\right)$ for the underlying model.

The redatumed virtual data (the Green's function $\mathbf{g}_{\mathbf{D}}$ ) can be retrieved in an optimization problem by minimizing the data misfits (Alkhalifah and Wu, 2016; Li et al., 2019):

$$
\min _{\mathbf{g}_{\mathbf{D}}} J=\frac{1}{2} \sum_{s}\left\|\left(\mathbf{u}\left(\mathbf{m}_{o}\right)+\mathbf{u}_{\mathbf{D}}\left(\mathbf{m}_{o}, \mathbf{g}_{\mathbf{D}}\right)\right)-\mathbf{d}\right\|_{2}^{2}
$$

where $\mathbf{d}$ is the observed seismic data, $\mathbf{m}_{o}$ denotes the overburden model and $\mathbf{u}$ and $\mathbf{u}_{\mathbf{D}}$ represent the simulated wavefield data at the receivers obtained by the acoustic wave equation 2 and the modeling equation 5, respectively. It is noteworthy that an estimate of the overburden model is required to account for the seismic wave propagation above the datum level. The gradient of the objective function with respect to the redatumed data $\left(\mathbf{g}_{\mathbf{D}}\right)$ is expressed as:

$$
\frac{\partial J}{\partial \mathbf{g}_{\mathbf{D}}}\left(\mathbf{x}_{v s}+\mathbf{h}, \mathbf{x}_{v s}, t\right)=-\sum_{s}\left\langle u\left(\mathbf{x}_{v s}, \mathbf{x}_{s}, t\right), u^{\dagger}\left(\mathbf{x}_{v s}+\mathbf{h}, \mathbf{x}_{s}, t\right)\right\rangle_{t}
$$


where, $u^{\dagger}$ is the backward propagating wavefield from the adjoint source at the receivers. We can compute the gradient for the virtual data $\left(\mathbf{g}_{\mathbf{D}}\right)$ by cross-correlating the forward and backward propagating wavefields at the datum level followed by a summation over the sources (Guo and Alkhalifah, 2019). Given a prior estimate of the overburden model, the redatuming process is performed iteratively using a gradient-based optimization algorithm. To some extent, the redatuming implementation is similar to the least-squares extended imaging (Symes, 2008), which can be related to earlier findings by Vasconcelos et al. (2010). We will apply the redatuming algorithm to the time-lapse seismic data to prepare the corresponding virtual datasets for the following target-oriented time-lapse full waveform inversion.

\section{Regularized TLFWI using deep learning}

Once the base and monitor datasets of the time-lapse experiment are redatumed to the datum level, just above the target zone, we then perform the double-difference waveform inversion (DDWI; Denli and Huang, 2009) to estimate the time-lapse changes in the monitoring (target) zone from the redatumed time-lapse datasets. In the DDWI algorithm, the baseline virtual dataset is first used to invert for the baseline model. Then, we update the monitor model starting from the inverted baseline model $\left(\mathbf{m}_{\text {ibase }}\right)$ by minimizing the misfits between the simulated time-lapse data difference for the virtual survey and the data difference of the monitor and baseline redatumed data. The double-difference objective function is expressed as:

$$
J_{d}(\mathbf{m})=\frac{1}{2}\left\|\mathbf{u}(\mathbf{m})-\mathbf{u}\left(\mathbf{m}_{\text {ibase }}\right)-\delta \mathbf{d}_{\text {virtual }}\right\|_{2}^{2},
$$


where, $\mathbf{u}$ is the simulated seismic data for the virtual survey using the acoustic modeling operator, $\mathbf{m}_{\text {ibase }}$ is the estimated baseline model, $\mathbf{m}$ is the monitor model and $\delta \mathbf{d}_{\text {virtual }}$ is the data difference for the virtual survey. The model updates $\left(\mathbf{m}-\mathbf{m}_{\text {ibase }}\right)$ are considered to represent the model changes ( $\delta \mathbf{m})$ in the target zone between monitor and baseline.

Prior information in the monitoring area from other geophysical surveys can help constrain or regularize the inverse problem to deliver a more accurate and robust estimate. Here, we assume that some well logs provide reliable measurements of the property changes in the monitoring zone, but have very limited coverage. To incorporate the prior information from well logs, a model-space regularization term is added to the objective function:

$$
J(\mathbf{m})=J_{d}(\mathbf{m})+\beta J_{m}(\mathbf{m})
$$

where,

$$
J_{m}(\mathbf{m})=\frac{1}{2}\left\|\mathbf{W}_{m}\left(\mathbf{m}-\left(\mathbf{m}_{\text {ibase }}+\delta \mathbf{m}_{\text {prior }}\right)\right)\right\|_{2}^{2}
$$

and $\beta$ is a weighting parameter balancing the contributions from the data and model misfit terms, $\mathbf{W}_{m}$ is a diagonal weighting matrix and $\delta \mathbf{m}_{\text {prior }}$ is a prior model for the property changes in the target zone that incorporates the well velocity information by deep learning.

\section{The prior model from DNN}

We derive the prior model by mapping the inverted time-lapse model to the interpreted facies from wells based on their statistical relationship predicted by a deep neural network (DNN) (Li et al., 2020a). The architecture of the DNN is shown in Figure 2. The neural network consists of input and output layers, with fully connected hidden layers in between. The data flows from the input layer, through the hidden layers, and to the output layer in a feedforward fashion. For a vector of inputs $\mathbf{a}_{0}$, a general forward- 
propagation equation is written as $\mathbf{a}_{l}=g_{l}\left(\mathbf{W}_{l} \mathbf{a}_{l-1}+\mathbf{b}_{l}\right)$, where $\mathbf{W}_{l}$ and $\mathbf{b}_{l}$ refer to the weighting matrix and the bias vector for the $l$ th layer, respectively. The activation function $g_{l}$ is used to induce nonlinearity in the formulation. Here, we adopt rectified linear unit (Relu) functions and a softmax activation function for the hidden layers and the output layer, respectively (Nair and Hinton, 2010). The softmax function admits a probability distribution that categorizes the input classification with regard to the output facies, as denoted by $\left(p_{1}, \cdots, p_{i}, \cdots, p_{n f}\right)$ in the output layer in Figure 2 .

Both the inverted baseline model $\left(\mathbf{m}_{\text {ibase }}\right)$ and the recovered velocity changes $(\delta \mathbf{m})$ from seismic data act as input features to discriminate the class of facies. Considering the property changes often occur in a limited depth range, the depth of the training samples is also used as an input feature to improve the prediction accuracy of the DNN-based facies classifier. We can identify at least two facies, representing the injection and non-injection areas, from the wells to set labels for the training dataset. In some complicated cases, the injection area needs to be described by more than one facies for a detailed interpretation.

To improve the performance of the trained DNN, the synthetic minority oversampling technique (SMOTE) is applied to enlarge the training datasets and reduce the imbalance of different facies (Chawla et al., 2002). We also employ a random dropout of 30\% to avoid over-fitting (Srivastava et al., 2014). For the training process, we initialize the weights and biases using a normal distribution and update them using the Adam optimizer, with the loss function given by a sparse cross entropy (Kingma and Ba, 2014). We set the batch size and the total number of epochs to 64 and 2,000, respectively, for training the network. Once the training process is finished, the DNN constructs a statistical connection between the features of the inverted model and the facies classes. We then apply the trained 
network to the target inversion zone to predict the facies distribution. Considering that the velocity changes for each of the facies have been defined from the well logs, the prior model for the velocity changes can be extracted from the facies distribution. Lastly, we implement a regularized DDWI by incorporating the prior model into the inversion workflow.

\section{The algorithm}

We implement the proposed target-oriented time-lapse full waveform inversion algorithm following the workflow shown in Figure 3:

(1) Using a reasonable overburden model, usually estimated from FWI, we redatum the baseline and monitor datasets from the Earth's surface to the new datum to provide the target-oriented time-lapse datasets.

(2) We then invert for the baseline model in the target zone ( $\left.\mathbf{m}_{\text {ibase }}\right)$ by matching the redatumed baseline datasets.

(3) We use the baseline inversion result $\left(\mathbf{m}_{\text {ibase }}\right)$ as a starting model to invert for the monitor model ( $\mathbf{m})$ using the data difference between monitor and baseline plus the synthetic data from $\mathbf{m}_{\text {ibase }}$. The model updates $\left(\delta \mathbf{m}=\mathbf{m}-\mathbf{m}_{\text {ibase }}\right)$ are considered as the estimated property changes.

(4) We then prepare the training data set for optimizing the DNN parameters: Label the selected training samples from the inverted model using the interpreted facies from the wells. Using the labeled data, we train the network.

(5) We apply the trained DNN to the target inversion zone and build the prior model for property changes. 
(6) We apply a similar FWI to that describes in (3), but based on the objective function defined in equation 9, which includes a regularization term given by the prior model.

(7) The resulting model changes represent the output of the approach.

\section{NUMERICAL EXAMPLES}

We apply the proposed inversion algorithm to the Marmousi and SEAM model examples. In the SEAM model example, the time-lapse dataset is provided by the SEAM project. In these two examples, we use an eighth-order in space finite difference scheme to simulate the seismic wave propagation. The absorbing boundary condition is employed on all sides of the model. We ignore the time-lapse changes in the overburden model and use the same overburden model to redatum the baseline and monitor data. We refer to Li et al. (2020b) for a detailed study of the impact of the overburden model and 4D noise on the process of redatuming and target-oriented time-lapse inversion.

\section{Marmousi synthetic example}

Figure 4a shows the baseline Marmousi model. We introduce velocity changes into the baseline model to construct the monitor model, and the position of these changes is highlighted by the red arrow in Figure 4a. We set the datum at depth $2.18 \mathrm{~km}$ (red dashed line in Figure 4a), just above the target monitoring zone. We only need to focus the inversion on a small area, indicated by the box in Figure 4a. Figure 4b shows the time-lapse velocity changes in the target region. A Ricker wavelet with peak frequency of $15 \mathrm{~Hz}$ is used as the source wavelet. We deploy 92 shots spread evenly at a depth of $50 \mathrm{~m}$ to excite 
the seismic wave energy, and use 461 receivers located at a depth $0.2 \mathrm{~km}$ to record the seismic data for the baseline and monitor models.

Starting from the initial model (Figure 5a), we invert for the overburden model above the datum using two frequency bands $3-12 \mathrm{~Hz}$ and $3-30 \mathrm{~Hz}$, sequentially. Figure $5 \mathrm{~b}$ shows the final inverted overburden model. Given the overburden model, we redatum the recorded baseline and monitor seismic data to the new datum to provide the virtual data sets for the following target-oriented inversion. The virtual shot gathers for the baseline at the datum level are shown in Figure 6a. Subtracting the baseline virtual data from the monitor virtual data, we obtain the time-lapse data difference for the virtual survey (Figure 6b). We first conduct a double-difference waveform inversion on the surface time-lapse seismic data, and only the target zone is updated for a fair comparison with the following inversion results. Figure 7a shows the DDWI result using the surface seismic data. Figure $7 \mathrm{~b}$ shows the target-oriented time-lapse velocity changes resolved from DDWI using the redatumed data. We can see that the target-oriented time-lapse inversion approach captures the velocity changes at the right position, and it shows better inversion accuracy than the timelapse inversion approach using the surface seismic data. However, the target-oriented DDWI result still includes some artifacts and the magnitude of the changes is underestimated. To preserve the sharp boundaries and suppress the artifacts in the DDWI result (Figure 7b), we also apply a TV regularization (Lin and Huang, 2015) to the targetoriented DDWI and the inversion result is shown in Figure 7c. We can see that the inversion result is improved, but still fails to describe the true velocity changes correctly especially below the depth of $2.85 \mathrm{~km}$. 
A vertical profile at $6.5 \mathrm{~km}$ (pointed by the white dashed line in Figure 4b) represents well log information, which provides complementary high-resolution information to the target zone. Two facies, representing the injection (non-zero velocity changes) and noninjection areas (zero velocity changes), are identified from the well and used as the labels for the training dataset. Once the training is finished, the deep neural network learns a statistical relationship between the inverted velocity and the facies. Then, we apply the trained neural network to the target inversion zone to predict the facies distribution, and derive the prior model for velocity changes (Figure 7d). At last, we conduct a regularized DDWI using the predicted prior model with the objective function shown in equation 9 . To suppress the artifacts in the non-injection area (no property changes), we penalize the relevant model misfits from the prior model more by increasing the weighting factor in the matrix $\mathbf{W}_{m}$ corresponding to the non-injection area. Specifically, $\mathbf{W}_{m}$ is not an identity matrix; the weighting factors for the non-injection and injection area are set to 100 and 1 , respectively. We consider a $20 \%$ misfit contribution from the model-space regularization term by adjusting the balancing factor $(\beta)$. Figure 7 e shows the estimated velocity changes from the regularized DDWI using the redatumed dataset. We can see that the image artifacts are alleviated and the inversion result is cleaner than that from the target-oriented DDWI with the TV regularization (Figure 7c). The shape of the recovered changes is similar to that of the prior model thanks to the constraints from the prior model. For a more detailed comparison, we plot the vertical profiles of the inversion results at location $6.8 \mathrm{~km}$ (Figure 8). The notch shown in the red line mainly comes from the model updates from the time-lapse seismic data. These figures show that the inversion result using the proposed 


\begin{abstract}
DL-based regularization scheme interprets the true velocity changes with higher accuracy and resolution compared to the other inversion results.
\end{abstract}

Figure 9 shows the predicted time-lapse data difference $\left(\mathbf{u}\left(\mathbf{m}_{\text {ibase }}+\delta \mathbf{m}\right)-\mathbf{u}\left(\mathbf{m}_{\text {ibase }}\right)\right)$ and the residuals $\left(\mathbf{u}\left(\mathbf{m}_{\text {ibase }}+\delta \mathbf{m}\right)-\mathbf{u}\left(\mathbf{m}_{\text {ibase }}\right)-\delta \mathbf{d}_{\text {virtual }}\right)$ using the estimated velocity changes $(\delta \mathbf{m})$ in Figure $7 \mathrm{~b}$ and $7 \mathrm{e}$. The predicted data difference matches the redatumed one in Figure $6 \mathrm{~b}$ to some extent, but there are still some residuals. These residuals are reasonable because the redatumed data difference includes some unpredictable artifacts generated from the linear optimization of the redatuming inverse problem using an imperfect overburden model. Besides, the residuals corresponding to the regularized inversion result are not reduced because the regularization renders a steady incorporation of the prior model into the inversion to compete with the contribution of redatumed time-lapse data.

\title{
Robustness of the DL-assisted prior model building
}

We add Gaussian white noise with various energy levels to the baseline and monitor data to test the robustness of the DL-assisted prior model building in the presence of random noise. The noisy shot gathers for the baseline with a signal-to-noise ratio (SNR) of $5.0 \mathrm{~dB}$ and $0.5 \mathrm{~dB}$ are shown in Figure $10 \mathrm{a}$ and $10 \mathrm{~b}$. The SNR is defined as $S N R_{d B}=10$ $\log _{10}\left(A_{\text {signal }}^{2} / A_{\text {noise }}{ }^{2}\right)$, where $A$ denotes root mean square (RMS) amplitude. We first conduct the target-oriented DDWI scheme following steps (1-3) in the workflow. Figure $10 \mathrm{c}$ and $10 \mathrm{~d}$ show the inverted baseline models in the target zone using the noisy data with a SNR of $5.0 \mathrm{~dB}$ and $0.5 \mathrm{~dB}$, respectively, and Figure 10e and 10f show the corresponding estimated velocity changes. As shown in Li et al. (2020b), the inverted baseline model 
$\left(\mathbf{m}_{\text {ibase }}\right)$ is less influenced by the random noise, while the estimated time-lapse changes $(\delta \mathbf{m})$ deteriorate as more noise are involved. $\mathbf{m}_{i b a s e}, \delta \mathbf{m}$ and the depth of the training samples are used as the input features of the DNN. Once the training is finished, the prior model can be predicted from the trained DNN. The prior model (Figures 10g) corresponding to the SNR of $5.0 \mathrm{~dB}$ is predicted well, while the prior model (Figures 10h) corresponding to the SNR of $0.5 \mathrm{~dB}$ is somewhat influenced by the seriously contaminated $\delta \mathbf{m}$. Overall, these test results abovementioned prove that the DL-assisted prior model building shows good robustness even with noisy data, because three input features, rather than only $\delta \mathbf{m}$, are combined to discriminate the facies classes.

\section{The SEAM model}

A 3D SEAM time-lapse model was constructed to simulate the changes in an oilfield reservoir. The model includes a water layer of $267.5 \mathrm{~m}$ depth on the top and four fault blocks across the reservoir with a thickness of about $500 \mathrm{~m}$. The provided seismic dataset were simulated by the SEAM project assuming an isotropic elastic medium and recorded by ocean-bottom nodes $(\mathrm{OBN})$. We extract a 2D line along the middle of the SEAM model from the OBN dataset to further verify the feasibility of the proposed method. Its acquisition survey geometry includes 60 OBNs with $175 \mathrm{~m}$ interval at $267.5 \mathrm{~m}$ depth and 501 shots with $25 \mathrm{~m}$ spacing at $10 \mathrm{~m}$ depth. A shot gather representing the pressure field for the baseline model is shown in Figure 11a. The corresponding time-lapse data difference are shown in Figure 11b. For simplification, as usually done with marine data, we ignore the elastic effects in the seismic data and use an acoustic assumption. 
Figure $12 \mathrm{a}$ and $12 \mathrm{~b}$ show the true baseline P-wave velocity and density model, respectively. Figure $12 \mathrm{c}$ and $12 \mathrm{~d}$ show the velocity and density changes in the target zone, caused by the hydrocarbon production. The time-lapse changes include both changes with sharp edges and smooth changes. In this case, we only invert for the velocity changes in the target zone. After production, both property changes and geometric shifts lead to the timelapse seismic response (Figure 11b).

The smoothed version of the average of the baseline and monitor models is used as the starting model (Figure 13), which potentially describes the kinematics of the medium accurately. Considering the target of interest is the reservoir between $3.5 \mathrm{~km}$ and $4.2 \mathrm{~km}$ depth, we set the datum at a depth $3 \mathrm{~km}$, which splits the whole model into the overburden and underlying model. Using the overburden from the starting model, we implement the redatuming process to retrieve the virtual datasets at the datum from the provided baseline and monitor datasets. Figure 14a shows the redatumed virtual shot gathers for baseline. The time-lapse difference between the redatumed monitor and baseline datasets is shown in Figure $14 \mathrm{~b}$. We then apply the DDWI algorithm to the redatumed data. Figures $15 \mathrm{a}$ and $15 \mathrm{~b}$ show the inverted baseline velocity and time-lapse velocity changes in the target zone, respectively.

Two pseudo wells at $7.5 \mathrm{~km}$ and $8.125 \mathrm{~km}$ (pointed by the white dashed line in Figure 12) are used to boost the model information, and three facies can be interpreted from the wells representing two injection levels and the none injection area. After training, the deep neural network is applied to the target inversion region to generate the prior model (Figure 15c). Considering that the smooth changes are not included well in the prior model, we use an identity weighting matrix $\mathbf{W}_{m}$ to admit smooth updates coming from the data misfits. 
We set the balancing factor $(\beta)$ to render a $40 \%$ misfit contribution from the model-space regularization term. The regularized DDWI method using the prior model provides a wellfocused inversion result (Figure 15d), which facilitates interpreting time-lapse variations in the reservoir. Note that the inverted model somewhat identified the two injection levels separated by a very thin none injection layer at $3.4 \mathrm{~km}$ depth. The vertical profiles at location $7.0 \mathrm{~km}$ (Figure 16), which was not used in the DNN training, also demonstrate that the inversion quality is enhanced using the proposed inversion algorithm.

\section{CONCLUSIONS}

We develop a regularized time-lapse full waveform inversion scheme focused on the desired target zone. The required target-oriented data are extracted from surface seismic data by waveform redatuming. In this case, the impact of the overburden complexity on the target inversion quality is mitigated, and the computational cost of the target area inversion is reduced. Considering that well logs provide detailed information for model changes with a limited coverage, we incorporate the well velocity information into the inversion by using an objective function that includes a model-space regularization term. The required prior model is predicted from a trained deep neural network, which identifies the statistical connection between the initial inverted model and facies interpreted from well logs. The numerical examples show that the proposed method improves the inversion resolution and accuracy with better consistency with well logs. 


\section{ACKNOWLEDGEMENTS}

We thank KAUST for its support. We also appreciate the seismic wave analysis group (SWAG) members for the fruitful discussions and helpful suggestions. The Shaheen supercomputing Laboratory in KAUST provides the computational support. 


\section{REFERENCES}

Alkhalifah, T., and Z. Wu, 2016, The natural combination of full and image-based waveform inversion: Geophysical Prospecting, 64, 19-30.

Alkhalifah, T., 2016, Full-model wavenumber inversion: An emphasis on the appropriate wavenumber continuation: Geophysics, 81, no. 3, R89-R98.

Alford, R.M., K.R. Kelly, and D.M. Boore, 1974, Accuracy of finite-difference modeling of the acoustic wave equation: Geophysics, 39, no. $6,834-842$.

Aragao, O., and P. Sava, 2020, Elastic full waveform inversion with probabilistic petrophysical clustering: Geophysical Prospecting, 68, no. 4, 1341-1355.

Arts, R., O. Eiken, A. Chadwick, P. Zweigel, L. Van der Meer, and B. Zinszner, 2003, Monitoring of $\mathrm{CO} 2$ injected at Sleipner using time lapse seismic data: 6th International Conference, Greenhouse Gas Control Technologies, 347-352.

Asnaashari, A., R. Brossier, S. Garambois, F. Audebert, P. Thore, and J. Virieux, 2013, Regularized seismic full waveform inversion with prior model information: Geophysics, 78, no. 2, R25-R36.

Asnaashari, A., R. Brossier, S. Garambois, F. Audebert, P. Thore, and J. Virieux, 2014, Time-lapse seismic imaging using regularized full-waveform inversion with a prior model: which strategy?: Geophysical prospecting, $\mathbf{6 3}$, no. 1, 78-98.

Ayeni, G., and B. Biondi, 2010, Target-oriented joint least-squares migration/inversion of time-lapse seismic data sets: Geophysics, 75, no. 3, R61-R73.

Bakulin, A., and R. Calvert, 2006, The virtual source method: Theory and case study: Geophysics, 71, no. 4, SI139-SI150. 
Biondi, E., B. Biondi, and G. Barnier, 2018, Target-oriented elastic full-waveform inversion through extended-migration redatuming: 88th Annual International Meeting, SEG, Expanded Abstracts, 1228-1232.

Borisov, D., and S.C. Singh, 2015, Three-dimensional elastic full waveform inversion in a marine environment using multicomponent ocean-bottom cables: a synthetic study: Geophysical Journal International, 201, no. 3, 1215-1234.

Chadwick, A., G. Williams, N. Delepine, V. Clochard, K. Labat, S. Sturton, M.L. Buddensiek, M. Dillen, M. Nickel, A.L. Lima, and R. Arts, 2010, Quantitative analysis of time-lapse seismic monitoring data at the Sleipner CO2 storage operation: The Leading Edge, 29, no. 2, 170-177.

Chawla, N.V., K.W. Bowyer, L.O. Hall, and W.P. Kegelmeyer, 2002, SMOTE: synthetic minority over-sampling technique: Journal of artificial intelligence research, 16, 321-357.

da Costa, C.A., J.C. Costa, W.E. Medeiros, D.J. Verschuur, and A.K. Soni, 2019, Targelevel waveform inversion: a prospective application of the convolution-type representation for the acoustic wavefield: Geophysical Prospecting, 67, no. 1, 69-84.

Denli, H., and L. Huang, 2009, Double-difference elastic waveform tomography in the time domain: 89th Annual International Meeting, SEG, Expanded Abstracts, 2302-2306.

Esser, E., L. Guasch, T. van Leeuwen, A.Y. Aravkin, and F.J. Herrmann, 2018, Total variation regularization strategies in full-waveform inversion: SIAM Journal on Imaging Sciences, 11, no. 1, 376-406.

Guo, Q., and T. Alkhalifah, 2019, Datum-based waveform inversion using a subsurfacescattering imaging condition: Geophysics, 84, no. 4, S251-S266. 
Guo, Q., and T. Alkhalifah, 2020, Target-oriented waveform redatuming and highresolution inversion: Role of the overburden: Geophysics, 85, no. 6, R525-R536.

Hicks, E., H. Hoeber, M. Houbiers, S.P. Lescoffit, A. Ratcliffe, and V. Vinje, 2016, Timelapse full-waveform inversion as a reservoir-monitoring tool-A North Sea case study: The Leading Edge, 35, no. 10, 850-858.

Kalita, M., V. Kazei, Y. Choi, and T. Alkhalifah, 2019, Regularized full-waveform inversion with automated salt flooding: Geophysics, 84, no. 4, R569-R582.

Kazei, V., and T. Alkhalifah, 2018, Time-lapse waveform inversion regularized by spectral constraints and Sobolev space norm: 88th Annual International Meeting, SEG, Expanded Abstracts, 5487-5491.

Kemper, M., and J. Gunning, 2014, Joint impedance and facies inversion — Seismic inversion redefined: First Break, 32, 89-95.

Kingma, D.P., and J. Ba, 2014, Adam: A method for stochastic optimization: arXiv preprint arXiv:1412.6980.

LeCun, Y., Y. Bengio, and G. Hinton, 2015, Deep learning: Nature, 521, no. 7553, 436444.

Li, Y., Y. Choi, T. Alkhalifah, Z. Li, and K. Zhang, 2018, Full-waveform inversion using a nonlinearly smoothed wavefield: Geophysics, 83, no. 2, R117-R127.

Li, Y., Q. Guo, Z. Li, and T. Alkhalifah, 2019, Elastic reflection waveform inversion with variable density: Geophysics, 84, no. 4, R553-R567.

Li, Y., T. Alkhalifah, and Z. Zhang, 2020a, High-resolution regularized elastic full waveform inversion assisted by deep learning: 82nd Annual International Meeting, EAGE, Expanded Abstracts, 1-5. 
Li, Y., Q. Guo, T. Alkhalifah, and V. Kazei, 2020b, Target-oriented time-lapse waveform inversion using redatumed data: Feasibility and robustness: 90th Annual International Meeting, SEG, Expanded Abstracts, 3783-3787.

Li, Y., T. Alkhalifah, and Q. Guo, 2020c, Target-oriented time-lapse waveform inversion using a deep learning-assisted regularization: 90th Annual International Meeting, SEG, Expanded Abstracts, 3744-3748.

Lin, Y., and L. Huang, 2015, Quantifying subsurface geophysical properties changes using double-difference seismic-waveform inversion with a modified total-variation regularization scheme: Geophysical Supplements to the Monthly Notices of the Royal Astronomical Society, 203, no. 3, 2125-2149.

Lumley, D.E., 2001, Time-lapse seismic reservoir monitoring: Geophysics, 66, no. 1, 5053.

Maharramov, M., B. L. Biondi, and M. A. Meadows, 2016, Time-lapse inverse theory with applications: Geophysics, 81, no. 6, R485-R501.

Nair, V., and G. E. Hinton, 2010, Rectified linear units improve restricted boltzmann machines: Proceedings of the 27th international conference on machine learning (ICML-10), 807-814.

Routh, P., R. Neelamani, R. Lu, S. Lazaratos, H. Braaksma, S. Hughes, R. Saltzer, J. Stewart, K. Naidu, H. Averill, and V. Gottumukkula, 2017, Impact of highresolution FWI in the Western Black Sea: Revealing overburden and reservoir complexity: The Leading Edge, 36, no. 1, 60-66.

Routh, P., G. Palacharla, I. Chikichev, and S. Lazaratos, 2012, Full wavefield inversion of time-lapse data for improved imaging and reservoir characterization: 82nd Annual 
International Meeting, SEG, Expanded Abstracts, 1-6, doi: 10.1190/segam20121043.1.

Singh, S., I. Tsvankin, and E.Z. Naeini, 2018, Bayesian framework for elastic fullwaveform inversion with facies information: The Leading Edge, 37, no. 12, 924 931.

Sirgue, L., and R.G. Pratt, 2004, Efficient waveform inversion and imaging: A strategy for selecting temporal frequencies: Geophysics, 69, no. 1, 231-248.

Song, C., T. Alkhalifah, and U.B. Waheed, 2021, Solving the frequency-domain acoustic VTI wave equation using physics-informed neural networks: Geophysical Journal International, 225, no. 2, 846-859.

Srivastava, N., G. Hinton, A. Krizhevsky, I. Sutskever, and R. Salakhutdinov, 2014, Dropout: a simple way to prevent neural networks from overfitting: The journal of machine learning research, 15, 1929-1958.

Symes, W.W., 2008, Migration velocity analysis and waveform inversion: Geophysical prospecting, 56, no. 6, 765-790.

Tarantola, A., 1984, Inversion of seismic reflection data in the acoustic approximation: Geophysics, 49, no. 8, 1259-1266.

Urosevic, M., R. Pevzner, A. Kepic, P. Wisman, V. Shulakova, and S. Sharma, 2010, Time-lapse seismic monitoring of $\mathrm{CO} 2$ injection into a depleted gas reservoirNaylor Field, Australia: The Leading Edge, 29, no. 2, 164-169.

van Manen, D.-J., J. O. A. Robertsson, and A. Curtis, 2007, Exact wave field simulation for finite-volume scattering problems: The Journal of the Acoustical Society of America, 122, EL115-EL121. 
Vasconcelos, I., P. Sava, and H. Douma, 2010, Nonlinear extended images via imagedomain interferometry: Geophysics, 75, no. 6, SA105-SA115

Wapenaar, K., and J. Fokkema, 2006, Green's function representations for seismic interferometry: Geophysics, 71, no. 4, SI33-SI46.

Wapenaar, K., and J. van der Neut, 2010, A representation for green function retrieval by multidimensional deconvolution: Journal Acoustical Society of America, 128, no. 6, EL366-EL371.

Yang, D., Y. Zheng, M. Fehler, and A. Malcolm, 2012, Target-oriented time-lapse waveform inversion using virtual survey: 82nd Annual International Meeting, SEG, Expanded Abstracts, 1-5, doi: 10.1190/segam2012-1308.1.

Yang, D., M. Meadows, P. Inderwiesen, J. Landa, A. Malcolm, and M. Fehler, 2015, Double-difference waveform inversion: Feasibility and robustness study with pressure data: Geophysics, 80, no. 6, M129-M141.

Yuan, S., N. Fuji, S. Singh, and D. Borisov, 2017, Localized time-lapse elastic waveform inversion using wavefield injection and extrapolation: 2-D parametric studies: Geophysical Journal International, 209, no. 3, 1699-1717.

Zhang, Z., and L. Huang, 2013, Double-difference elastic-waveform inversion with prior information for time-lapse monitoring: Geophysics, 78, no. 6, R259-R273.

Zhang, Z.D., T. Alkhalifah, E.Z. Naeini, and B. Sun, 2018, Multiparameter elastic full waveform inversion with facies-based constraints: Geophysical Journal International, 213, no. 3, 2112-2127.

Zhang, Z., and T. Alkhalifah, 2019, Regularized elastic full waveform inversion using deep learning: Geophysics, 84, no. 5, 1-47. 
Zhao, Y., F. Niu, H. Liu, X. Jia, J. Yang, and S. Huo, 2020, Source-receiver interferometric redatuming using sparse buried receivers to address complex near-surface environments: A case study of seismic imaging quality and time-lapse repeatability: Journal of Geophysical Research: Solid Earth, 125, no. 6, e2020JB019496.

Zheng, Y., P. Barton, and S. Singh, 2011, Strategies for elastic full waveform inversion of time-lapse ocean bottom cable (OBC) seismic data: 81st Annual International Meeting, SEG, Expanded Abstracts, 4195-4200. 


\section{LISTS OF FIGURES}

1 Diagram demonstration for the redatuming operation. The sources $\left(\mathbf{x}_{s}\right)$ and receivers $\left(\mathbf{x}_{r}\right)$ for a typical acquisition survey are located near the earth surface. The redatuming is applied to redatum the surface seismic data from the earth surface to the datum level. The virtual sources $\left(\mathbf{x}_{v s}\right)$ and receivers $\left(\mathbf{x}_{v r}\right)$ for the redatumed dataset are deployed at the datum level (red line). $\mathbf{x}_{v r}=\mathbf{x}_{v s}+\mathbf{h}$ ( $\mathbf{h}:$ the subsurface offset vector). The wavefield distortions caused by the complex overburden is desired to be removed by the redatuming implementation.

2 The architecture of the Deep Neural Network. The DNN consists of input and output layers, with fully connected hidden layers in between. There are three hidden layers (denoted as $L_{1}, L_{2}, L_{3}$ ) and 64 neurons (denoted as $\left.N_{1}, \cdots, N_{i, \cdots,} N_{64}\right)$ in each layer. Three input features for the input layer include the inverted baseline model $\left(\mathbf{m}_{\text {ibase }}\right)$, the inverted property changes $(\delta \mathbf{m})$ and the depth. The output layer delivers the probabilities $\left(p_{1}, \cdots, p_{i}, \cdots, p_{n f}\right)$ of being categorized into each facies given the input.

3 The workflow for the proposed inversion method. The input and output for the workflow are shown in the blue boxes; The DDWI part is described by the red boxes; The DL part for building the prior model is shown by the black boxes; The dashed line indicates that it is activated according to the inversion requirement, and for this case, the two dashed lines pointing to "DNN-based facies classification" are activated after the first 
implementation of DDWI, and the dashed line pointing to "Output the model changes" is activated after including regularization into another implementation of DDWI.

4 (a) The true baseline model and (b) the true time-lapse velocity changes in the target zone for the Marmousi example. The white dashed line shows the well location.

5 (a) The initial model and (b) the inverted overburden model using standard FWI for the Marmousi example.

6 (a) The virtual shot gathers for baseline at the datum level and (b) the time-lapse data difference ( $\delta \mathbf{d}_{\text {virtual }}$ ) for the virtual survey for the Marmousi example.

7 The Marmousi example: (a) the DDWI result using the surface seismic data (only the target zone is updated for a fair comparison), (b) the target-oriented DDWI result using the redatumed data, (c) the target-oriented DDWI result using a TV regularization, (d) the predicted prior model using deep learning, (e) the final inverted velocity changes regularized by the prior model in Figure 7d.

8 The vertical profiles of the velocity changes at $\mathrm{x}=6.8 \mathrm{~km}$ for the Marmousi example. Green line: the DDWI result using the surface seismic data shown in Figure 7a; blue line: the target-oriented DDWI result shown in Figure 7b; magenta line: the targetoriented DDWI result using a TV regularization shown in Figure 7c; red line: the final inversion result using DL-assisted regularization shown in Figure 7e; black dashed line: the true velocity changes.

9 The Marmousi example: (a) The predicted time-lapse data difference $\left(\mathbf{u}\left(\mathbf{m}_{\text {ibase }}+\delta \mathbf{m}\right)-\mathbf{u}\left(\mathbf{m}_{\text {ibase }}\right)\right)$ and (b) the residuals $\left(\mathbf{u}\left(\mathbf{m}_{\text {ibase }}+\delta \mathbf{m}\right)-\mathbf{u}\left(\mathbf{m}_{\text {ibase }}\right)-\delta \mathbf{d}_{\text {virtual }}\right)$ for the virtual survey using the inverted velocity changes $(\delta \mathbf{m})$ in Figure $7 \mathrm{~b}$; (c) the predicted 
time-lapse data difference and (d) the residuals corresponding to the inverted velocity changes in Figure 7e. (These figures are plotted in the same scale.)

10 The robustness test of the DL-assisted prior model building using the Marmousi example: (a-b) The noisy shot gathers for the baseline with an SNR of $5.0 \mathrm{~dB}$ and $0.5 \mathrm{~dB}$; (c-d) The inverted baseline models, (e-f) the recovered time-lapse changes and (g-h) the predicted prior model in the target zone corresponding to the noisy data shown in Figures $10(a-b)$

11 (a) A shot gather of the pressure component for the baseline model and (b) the corresponding time-lapse data difference for the SEAM example.

12 The baseline SEAM model: (a) P-wave velocity and (b) density. The true timelapse (c) velocity and (d) density changes in the target zone for the SEAM example. (The white dashed lines denote the pseudo wells at $7.5 \mathrm{~km}$ and $8.125 \mathrm{~km}$ )

13 The starting model for the SEAM example: a smoothed version of the average of the baseline and monitor models, (a) velocity and (b) density.

14 The virtual shot gathers for baseline at the datum level and (b) the time-lapse data difference for the virtual survey for the SEAM example.

15 The SEAM example: (a) the inverted baseline model in the target zone, (b) the standard target-oriented time-lapse inversion result, (c) the predicted prior model using deep learning, (d) the final inverted velocity changes regularized by the prior model in Figure 15c.

16 The vertical profiles of the inverted and true velocity changes at $\mathrm{x}=7.0 \mathrm{~km}$ for the SEAM example. Blue line: the standard target-oriented DDWI result; red line: the final 
inversion result using DL-assisted regularization; black dashed line: the true velocity changes. 


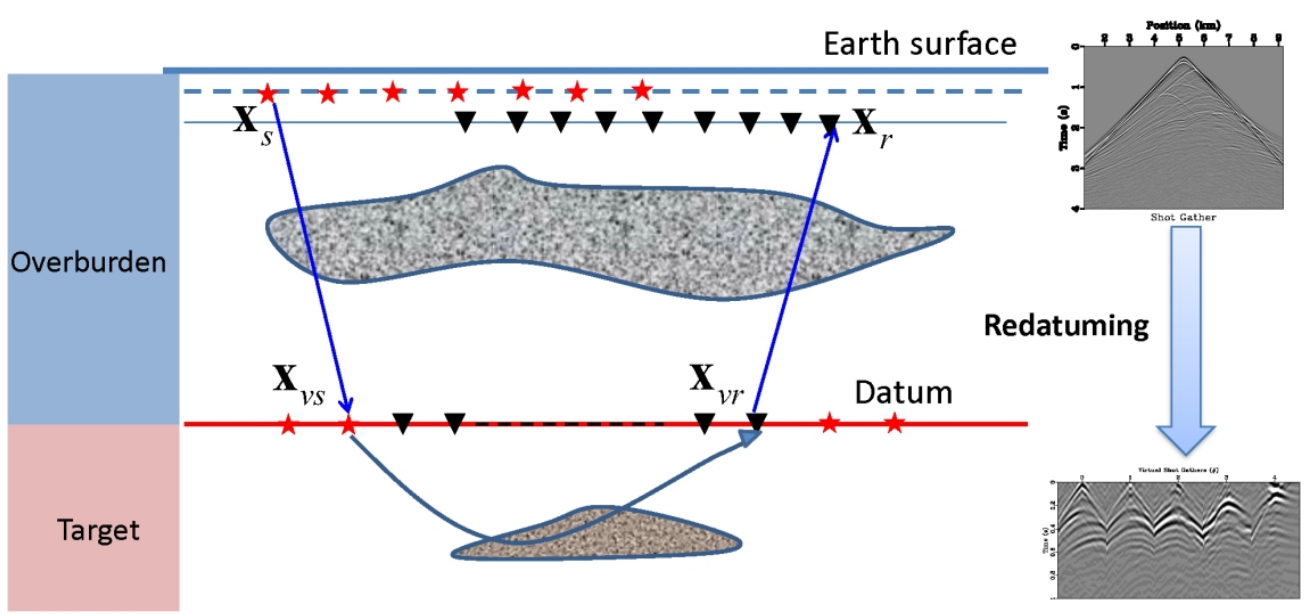

Figure 1. Diagram demonstration for the redatuming operation. 


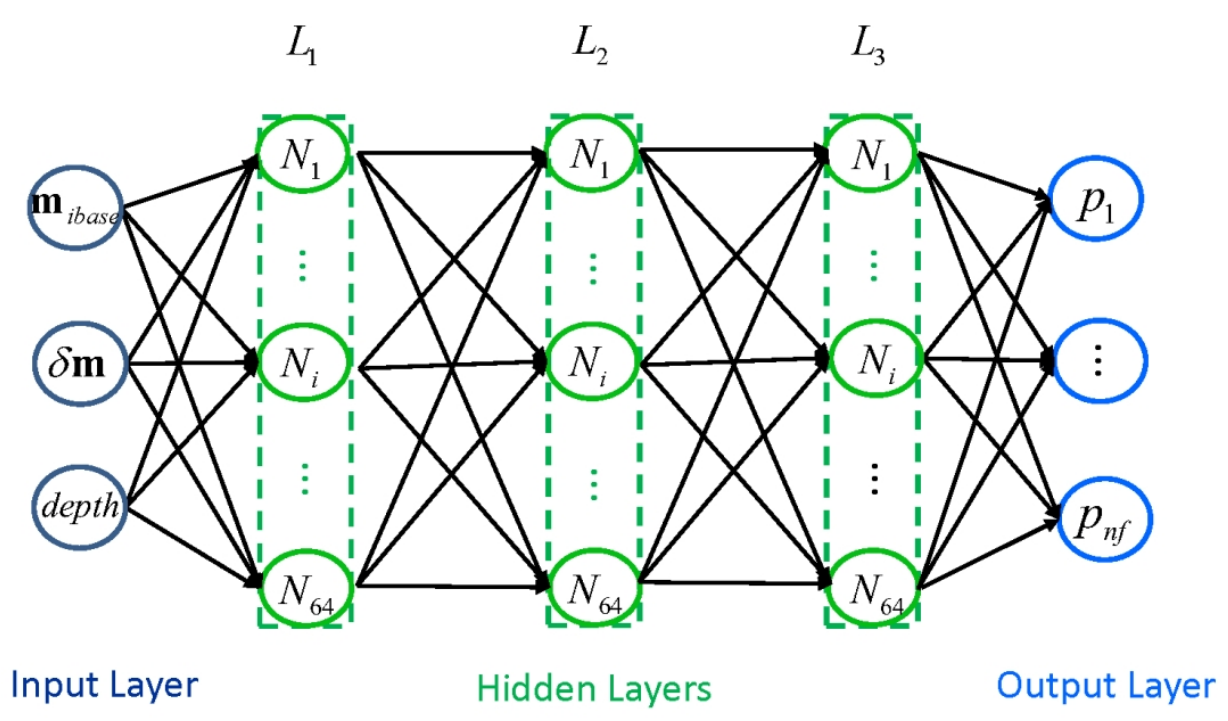

Figure 2. The architecture of the Deep Neural Network. 


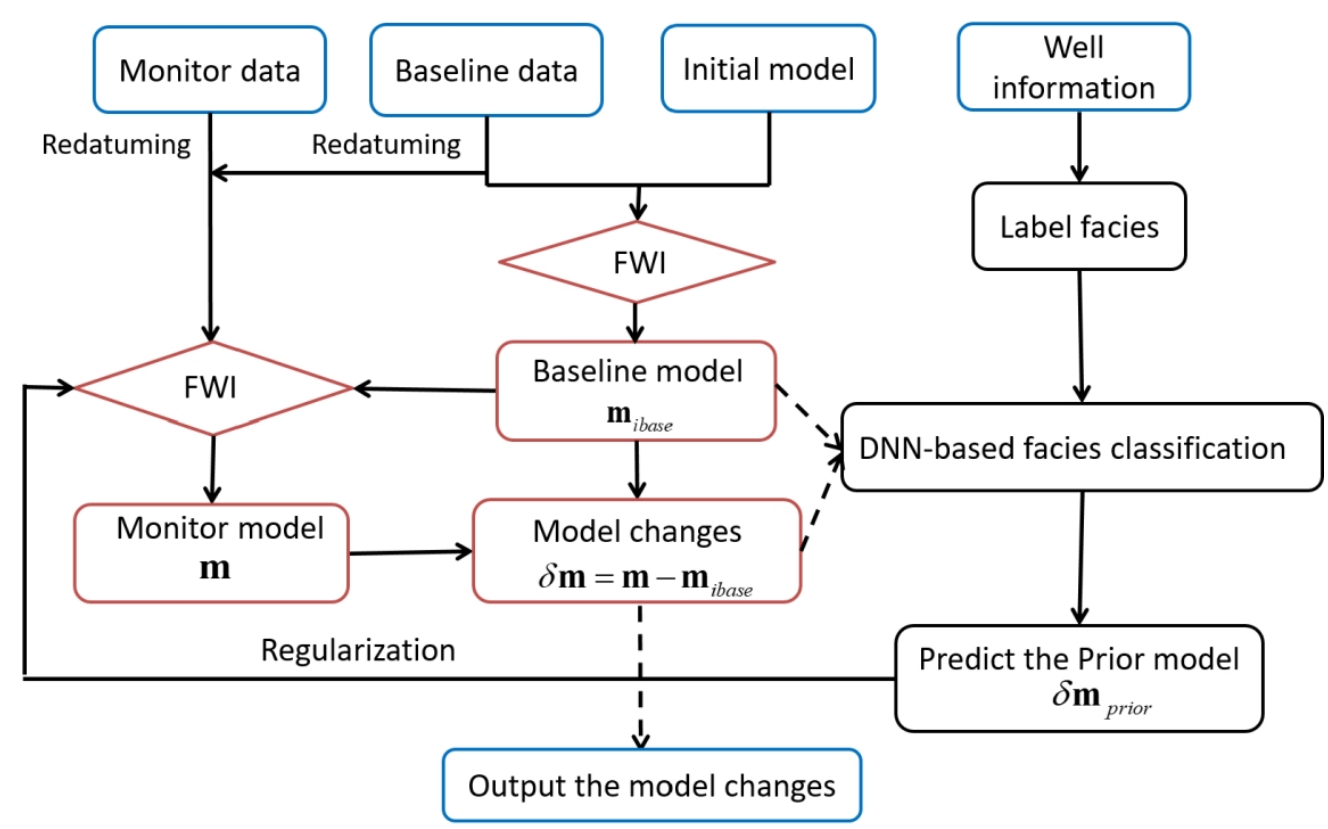

Figure 3. The workflow for the proposed inversion method. 


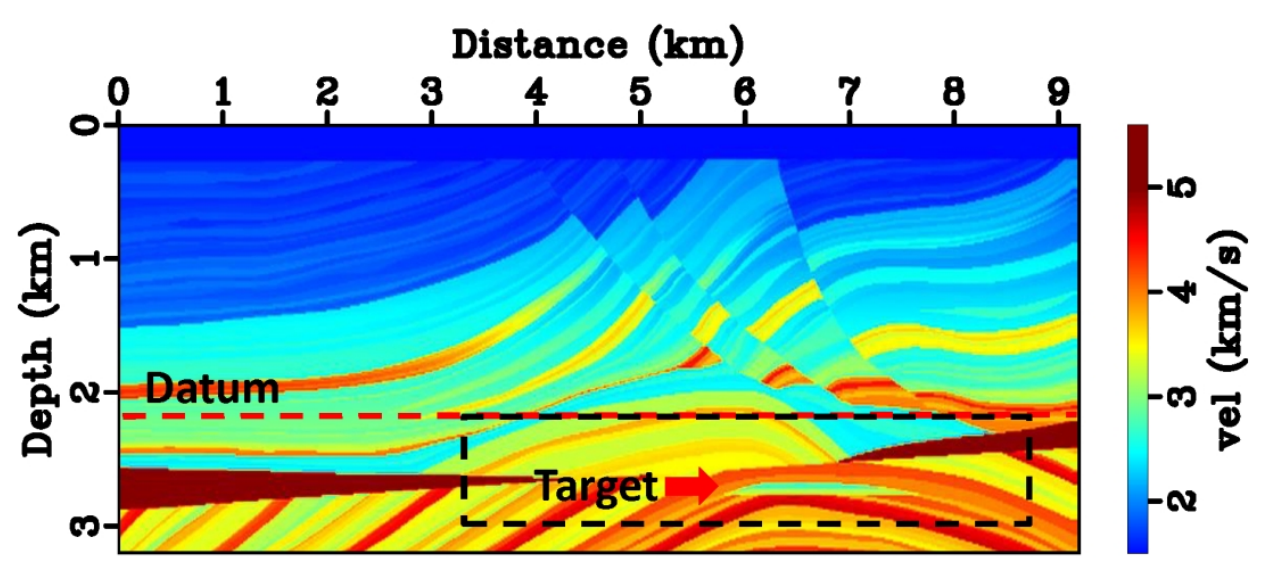

Figure 4. (a) The true baseline model 


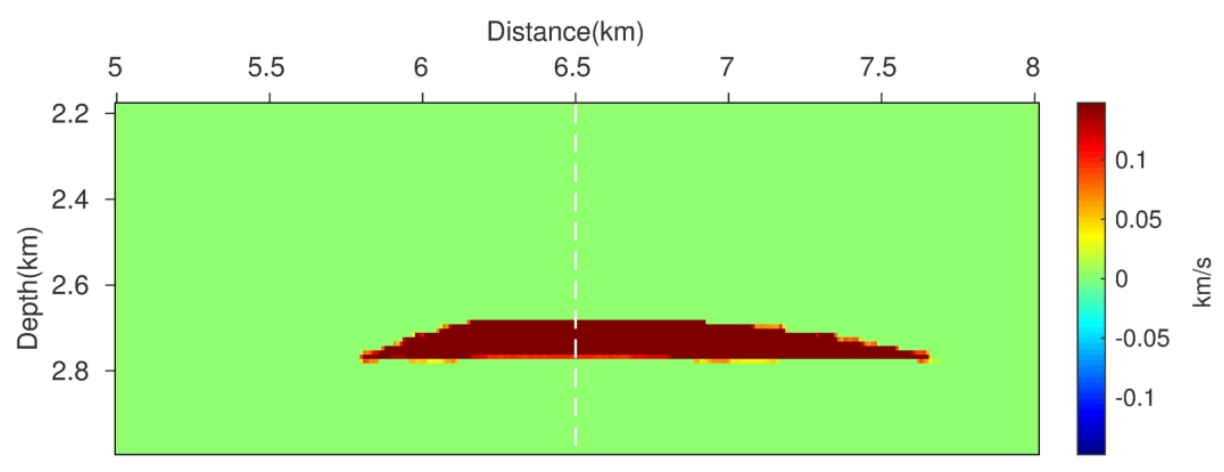

Figure 4. (b) The true time-lapse velocity changes in the target zone for the Marmousi example. The white dashed line shows the well location.

$188 \times 71 \mathrm{~mm}(300 \times 300$ DPI) 


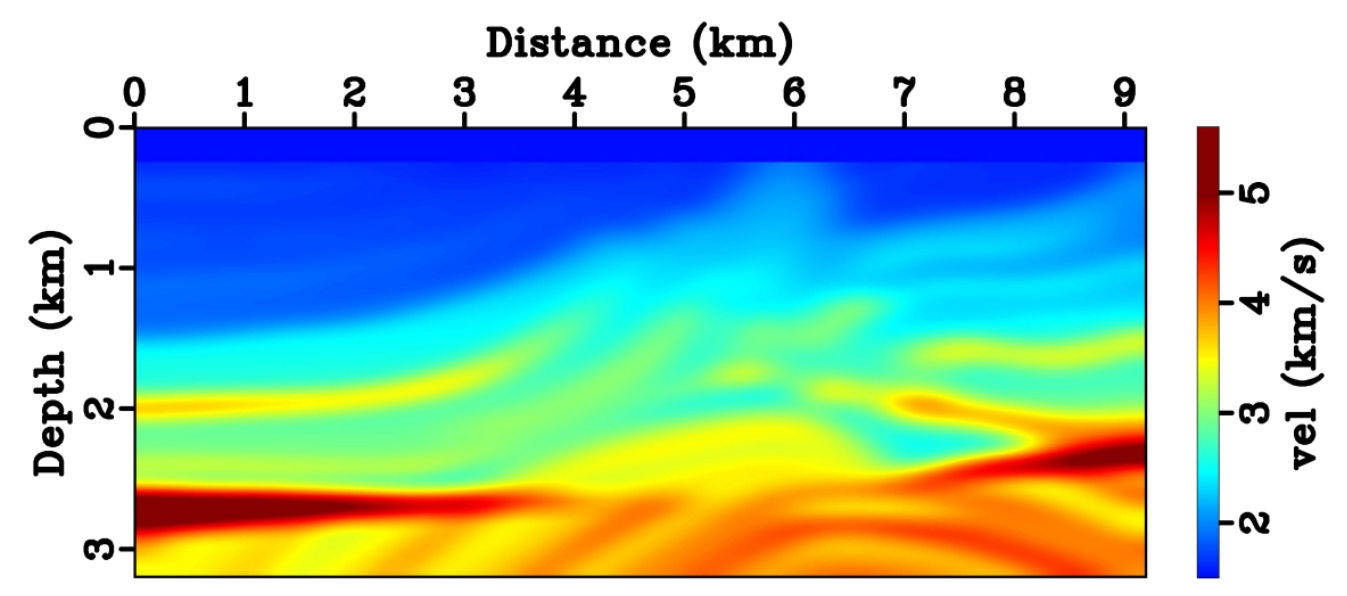

Figure 5. (a) The initial model

$549 \times 239 \mathrm{~mm}(300 \times 300$ DPI) 


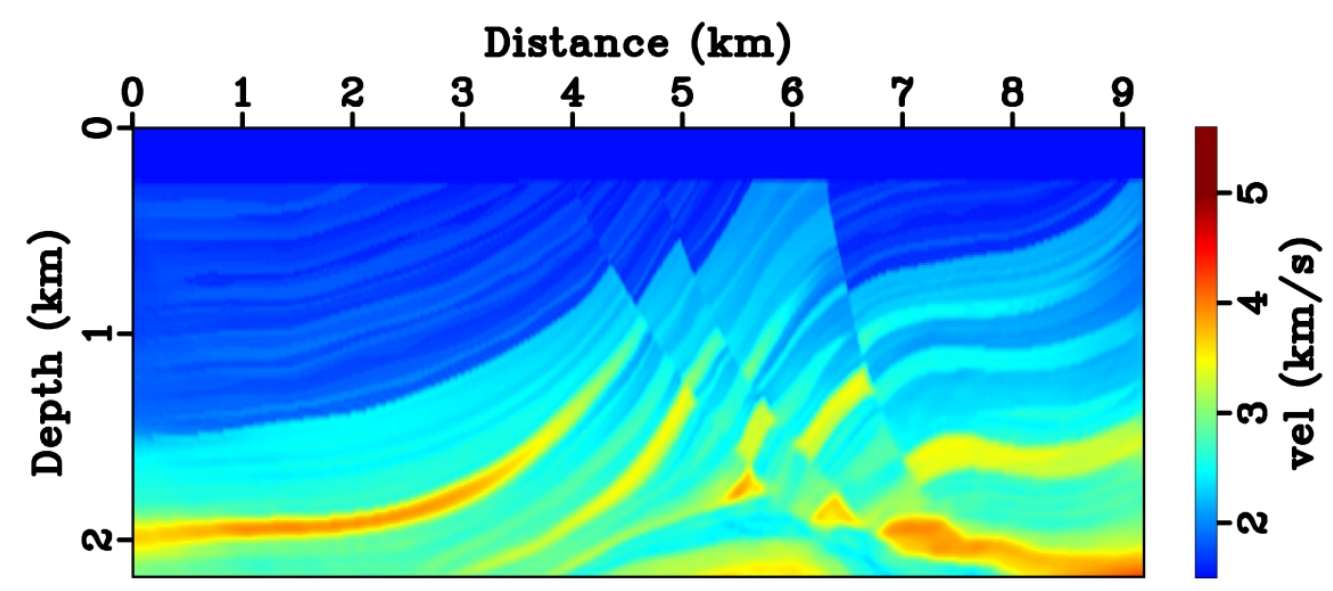

Figure 5. (b) The inverted overburden model using standard FWI for the Marmousi example.

$549 \times 239 \mathrm{~mm}(300 \times 300 \mathrm{DPI})$ 


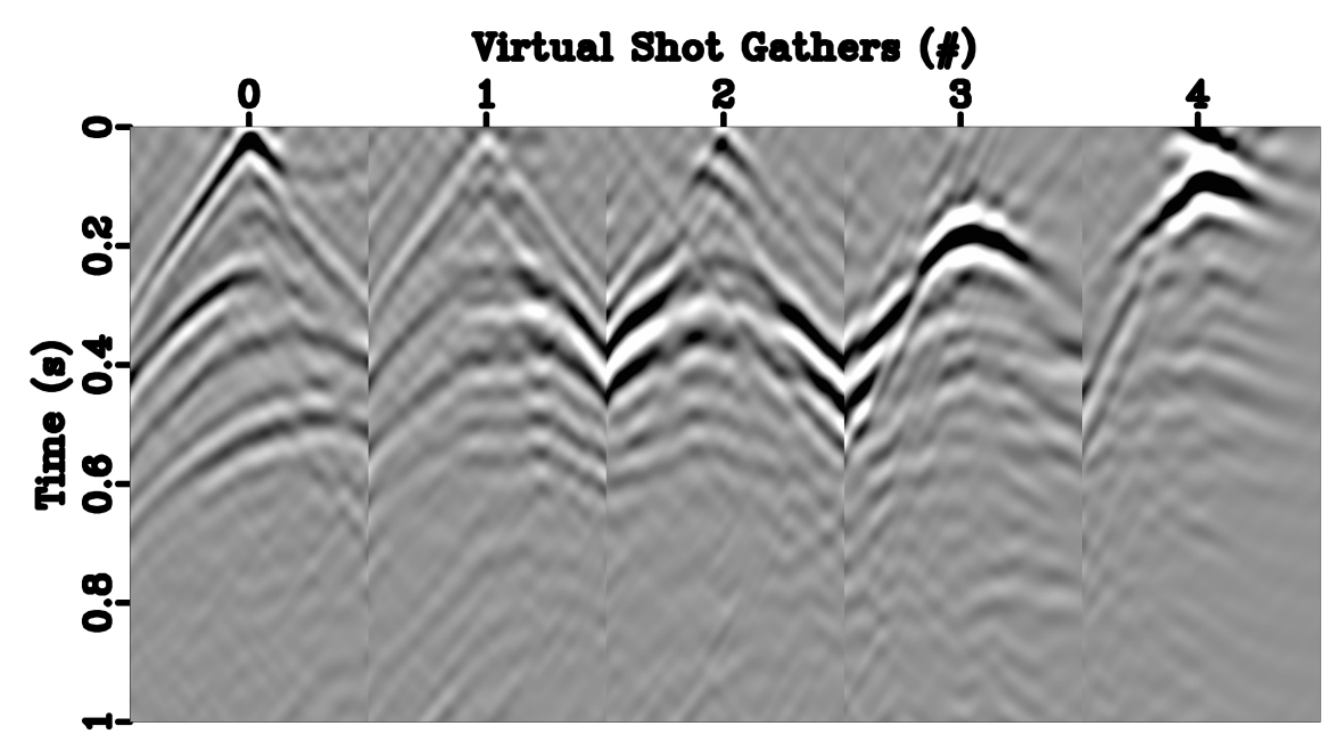

Figure 6. (a) The virtual shot gathers for baseline at the datum level

$417 \times 227 \mathrm{~mm}(300 \times 300 \mathrm{DPI})$ 


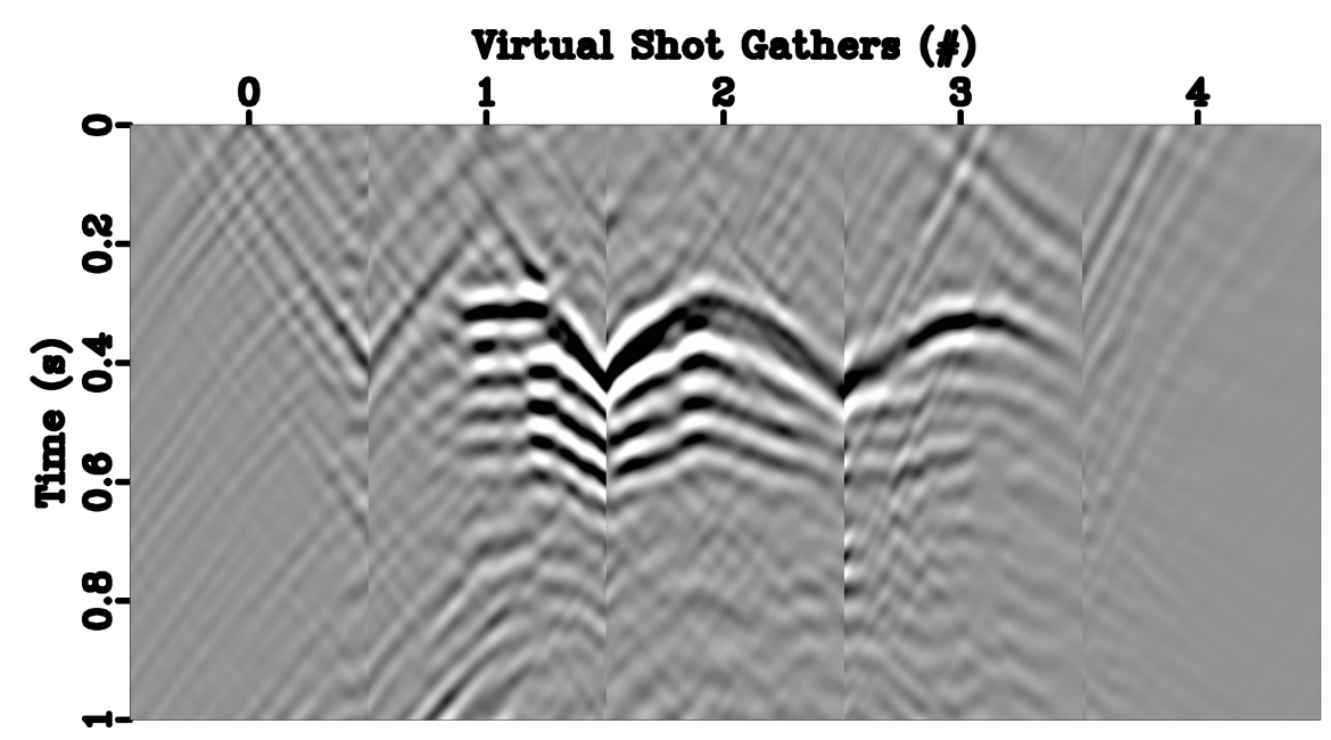

Figure 6. (b) the time-lapse data difference for the virtual survey for the Marmousi example. $417 \times 227 \mathrm{~mm}(300 \times 300$ DPI $)$ 


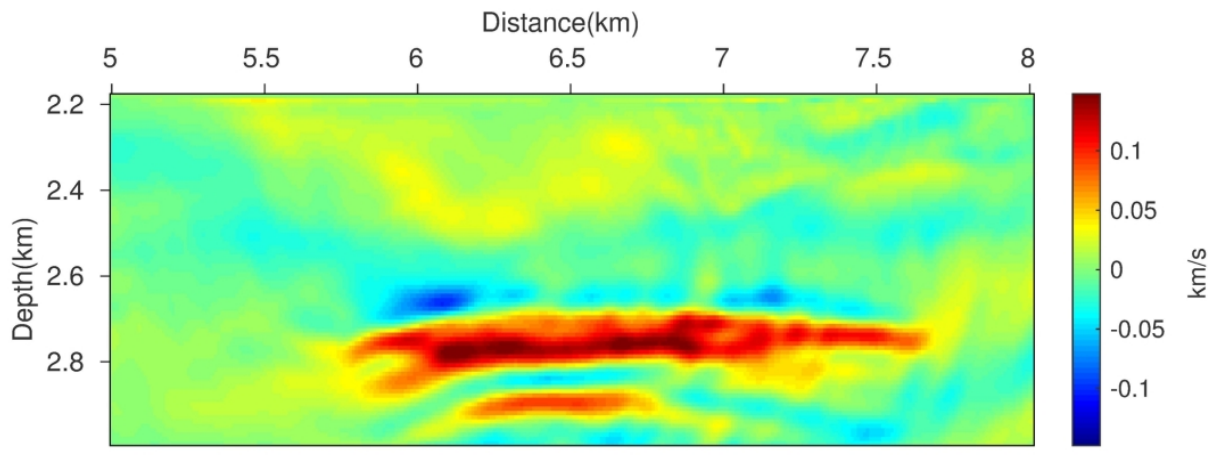

Figure 7. The Marmousi example: (a) the DDWI result using the surface seismic data (only the target zone is updated for a fair comparison)

$188 \times 71 \mathrm{~mm}(300 \times 300 \mathrm{DPI})$ 


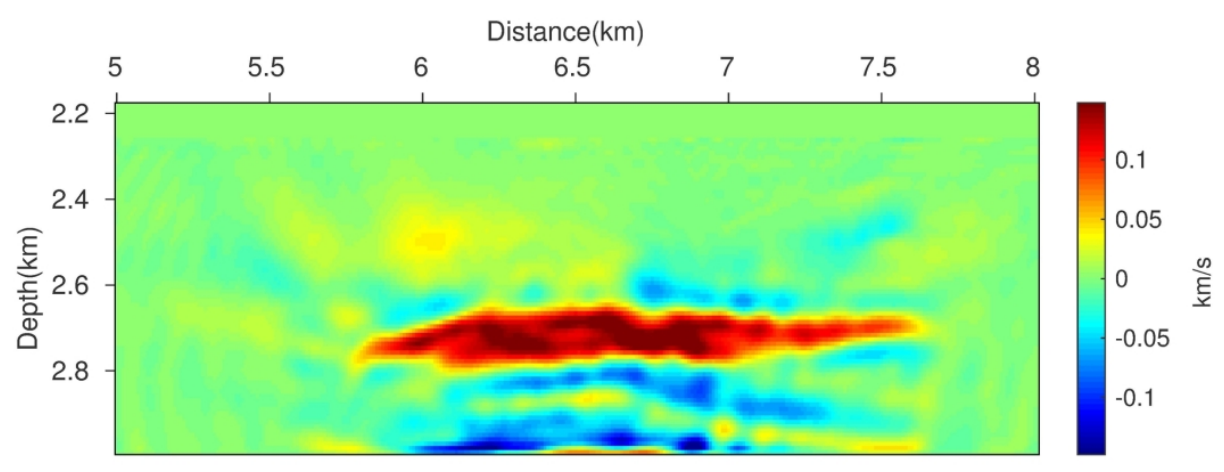

Figure 7. (b) the target-oriented DDWI result using the redatumed data $188 \times 71 \mathrm{~mm}(300 \times 300 \mathrm{DPI})$ 


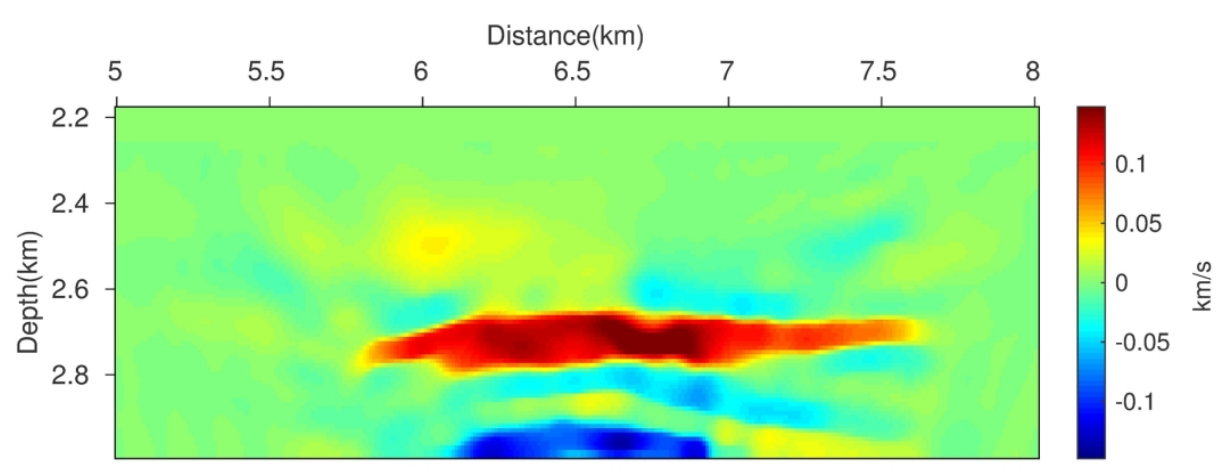

Figure 7. (c) the target-oriented DDWI result using a TV regularization $188 \times 71 \mathrm{~mm}(300 \times 300 \mathrm{DPI})$ 


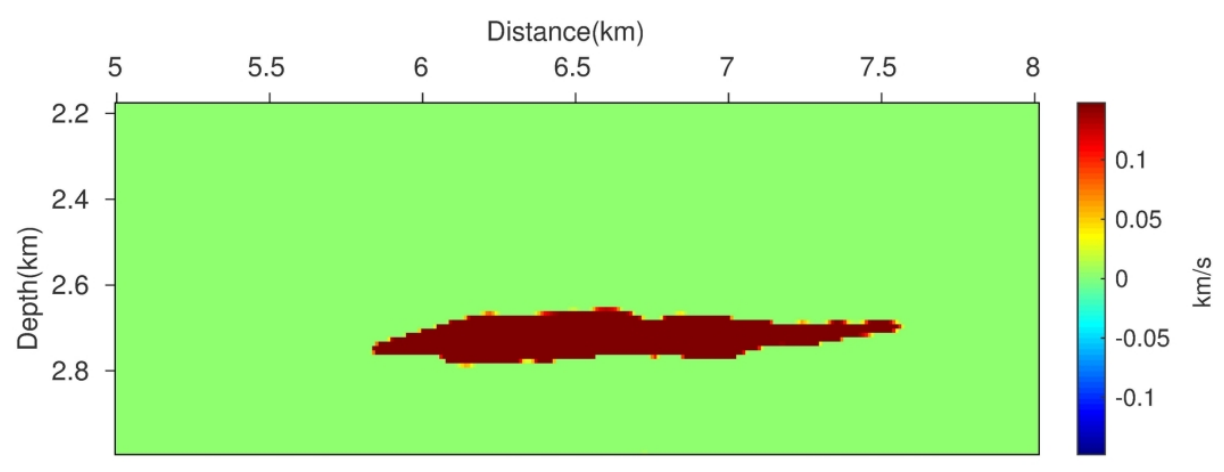

Figure 7. (d) the predicted prior model using deep learning $188 \times 71 \mathrm{~mm}(300 \times 300$ DPI $)$ 


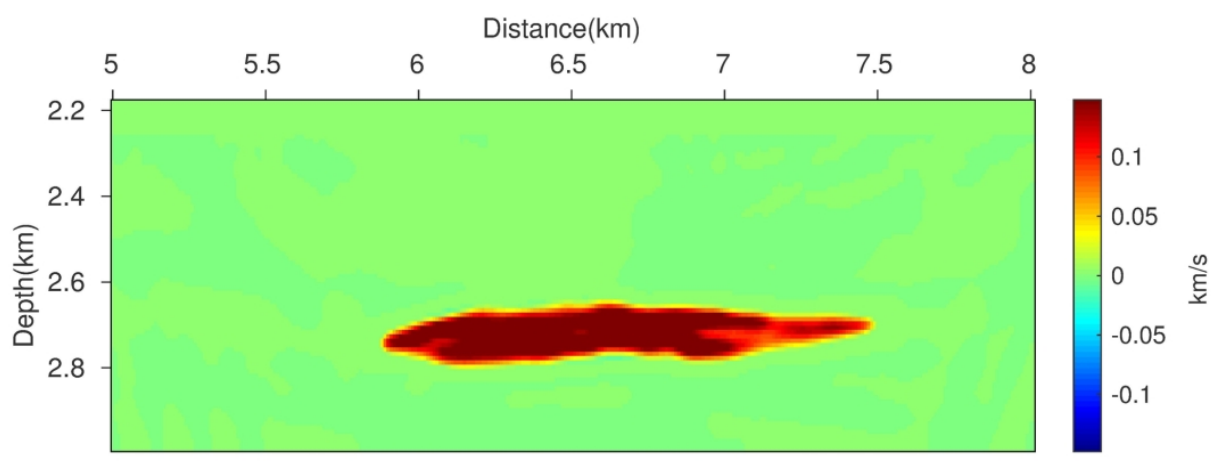

Figure 7. (e) the final inverted velocity changes regularized by the prior model in Figure 7d. $188 \times 71 \mathrm{~mm}(300 \times 300 \mathrm{DPI})$ 


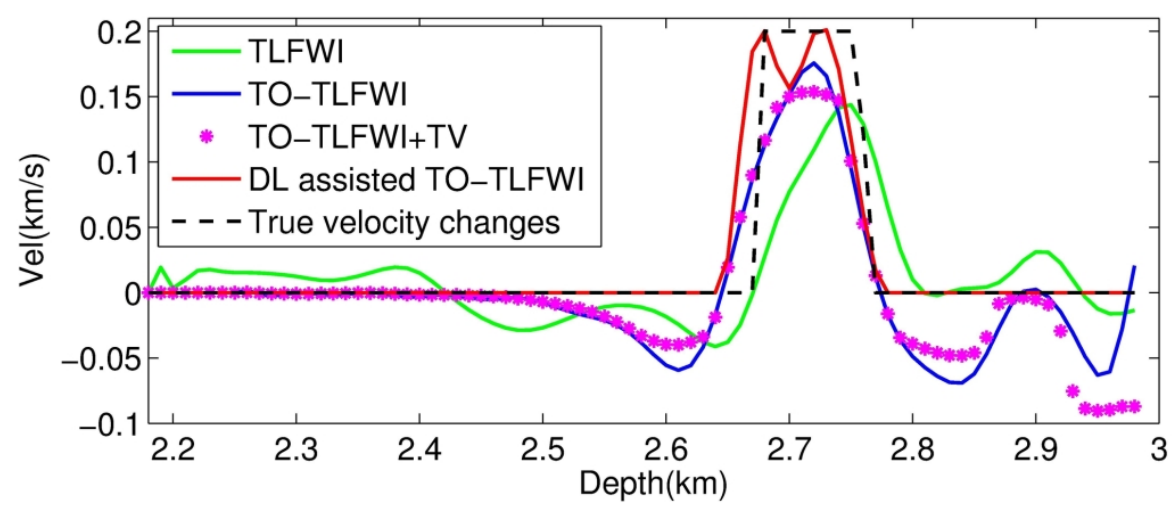

Figure 8. The vertical profiles of the velocity changes at $x=6.8 \mathrm{~km}$ for the Marmousi example.

$199 \times 80 \mathrm{~mm}(300 \times 300 \mathrm{DPI})$ 


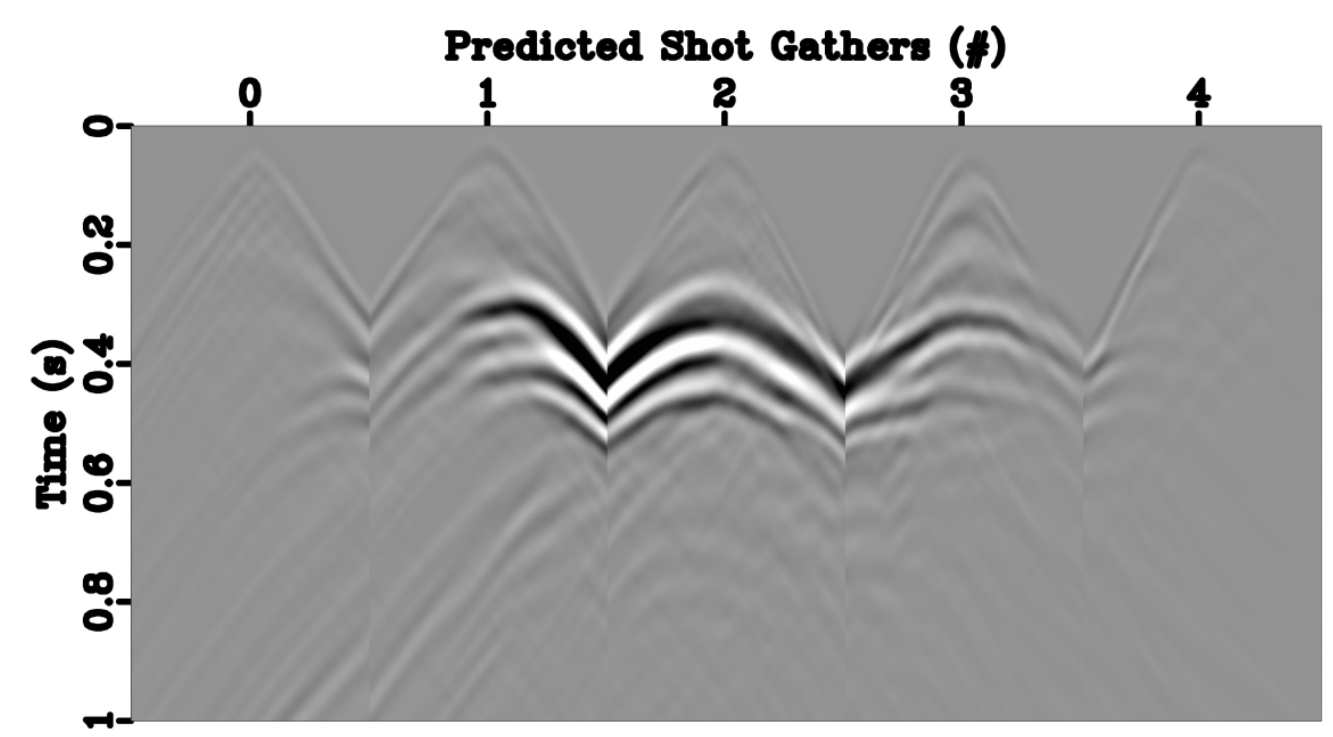

Figure 9. The Marmousi example: (a) The predicted time-lapse data difference for the virtual survey using the inverted velocity changes in Figure $7 \mathrm{~b}$

$417 \times 227 \mathrm{~mm}(300 \times 300$ DPI $)$ 


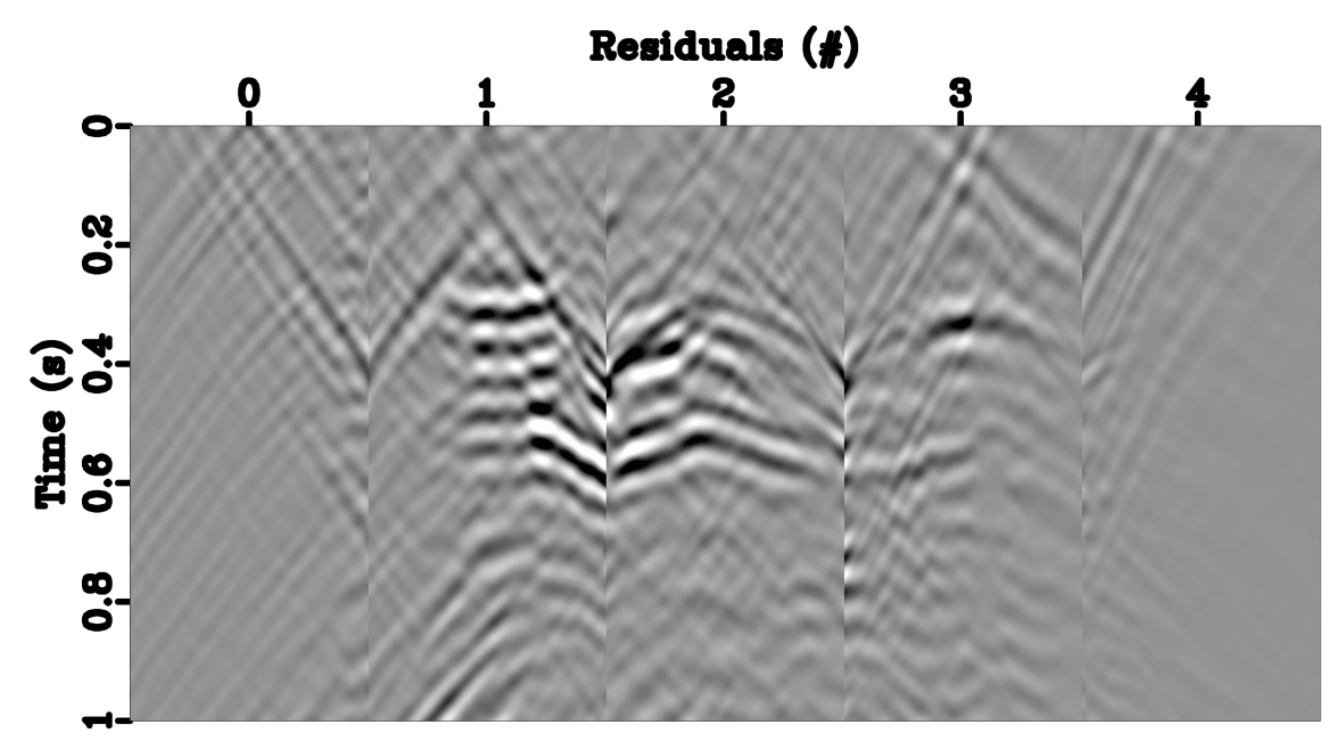

Figure 9. (b) the residuals for the virtual survey using the inverted velocity changes in Figure 7b $417 \times 227 \mathrm{~mm}(300 \times 300 \mathrm{DPI})$ 


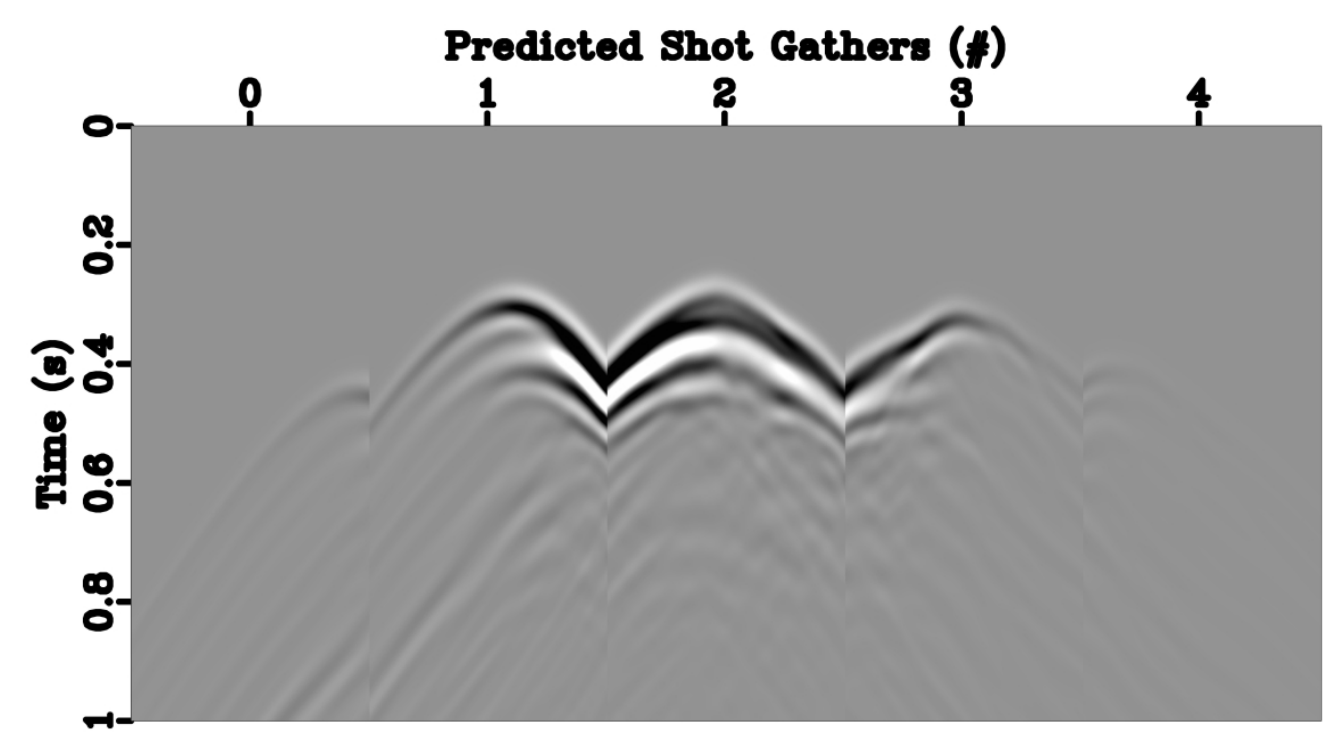

Figure 9. (c) the predicted time-lapse data difference corresponding to the inverted velocity changes in Figure 7e.

$417 \times 227 \mathrm{~mm}(300 \times 300 \mathrm{DPI})$ 


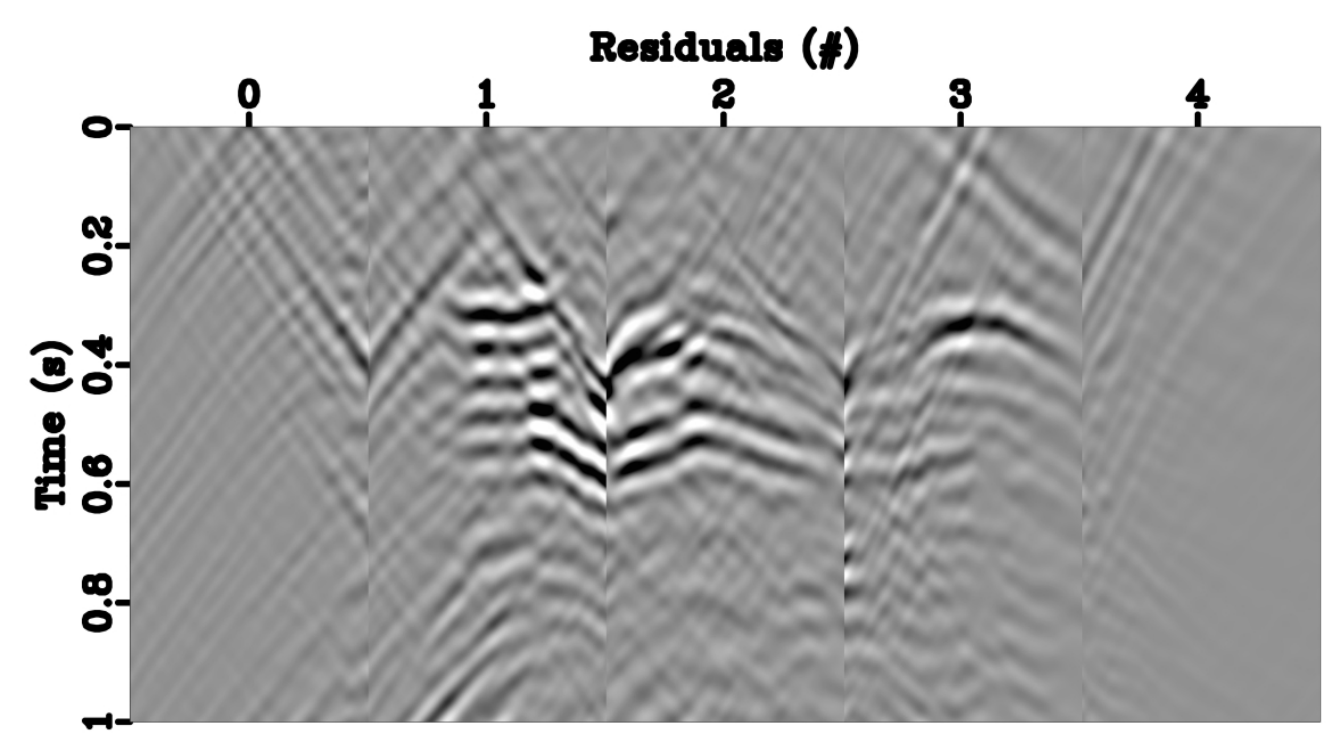

Figure 9. (d) the residuals corresponding to the inverted velocity changes in Figure 7e. $417 \times 227 \mathrm{~mm}(300 \times 300$ DPI $)$ 


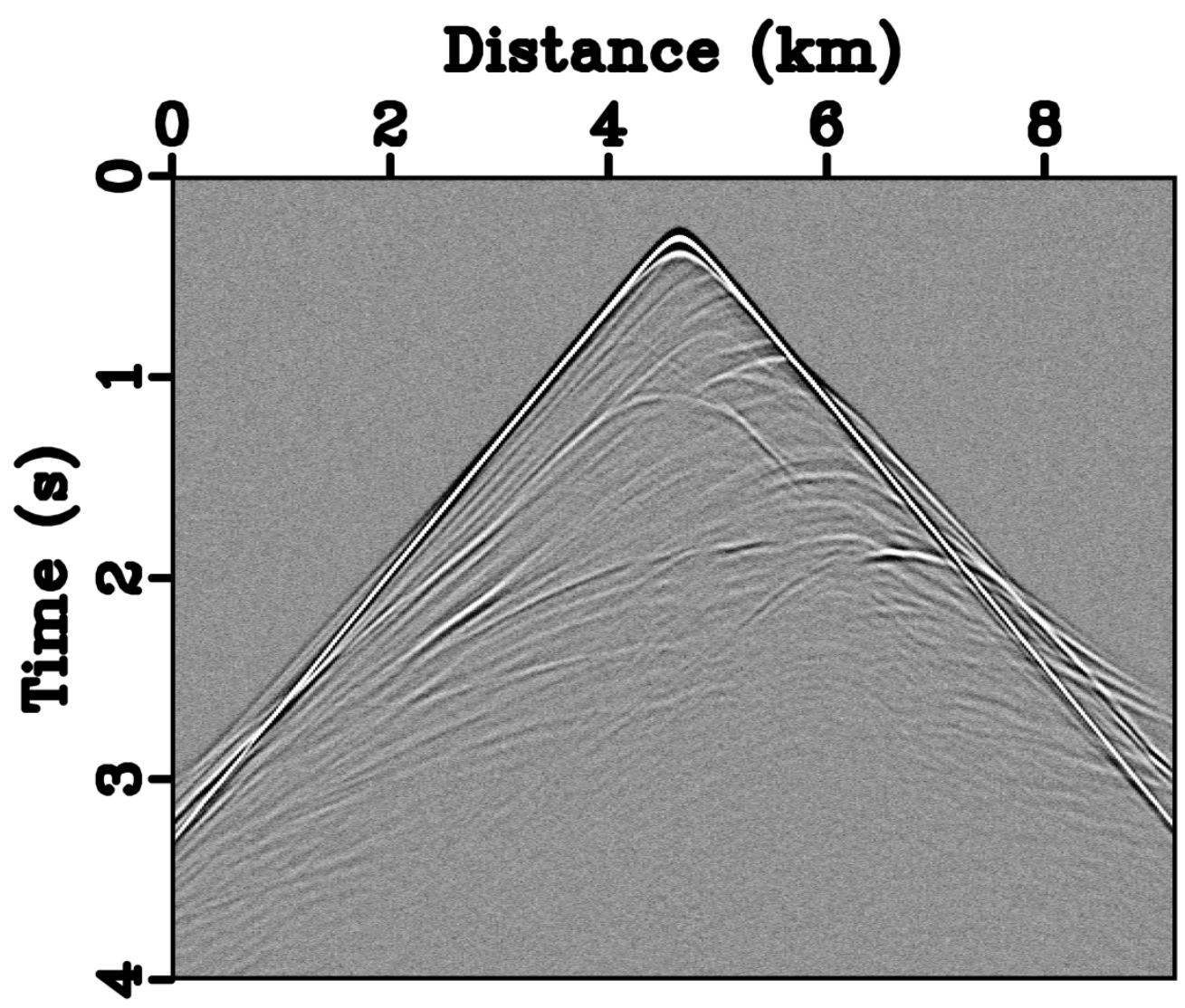

Figure 10. The robustness test of the DL-assisted prior model building using the Marmousi example: (a) The noisy shot gather for the baseline with an SNR of $5.0 \mathrm{~dB}$;

$286 \times 240 \mathrm{~mm}(300 \times 300 \mathrm{DPI})$ 


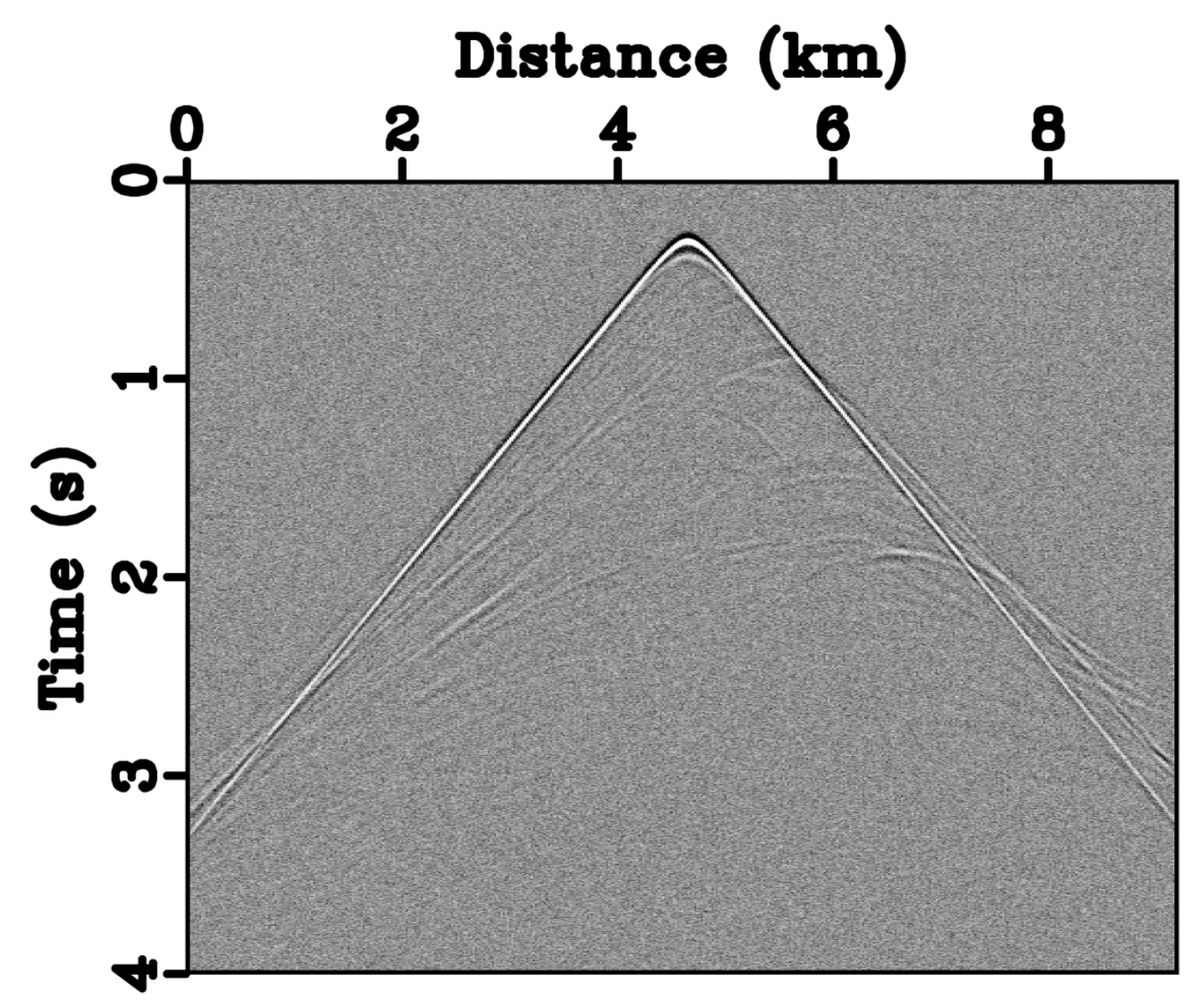

Figure 10. (b) The noisy shot gather for the baseline with an SNR of $0.5 \mathrm{~dB}$; $286 \times 240 \mathrm{~mm}(300 \times 300 \mathrm{DPI})$ 


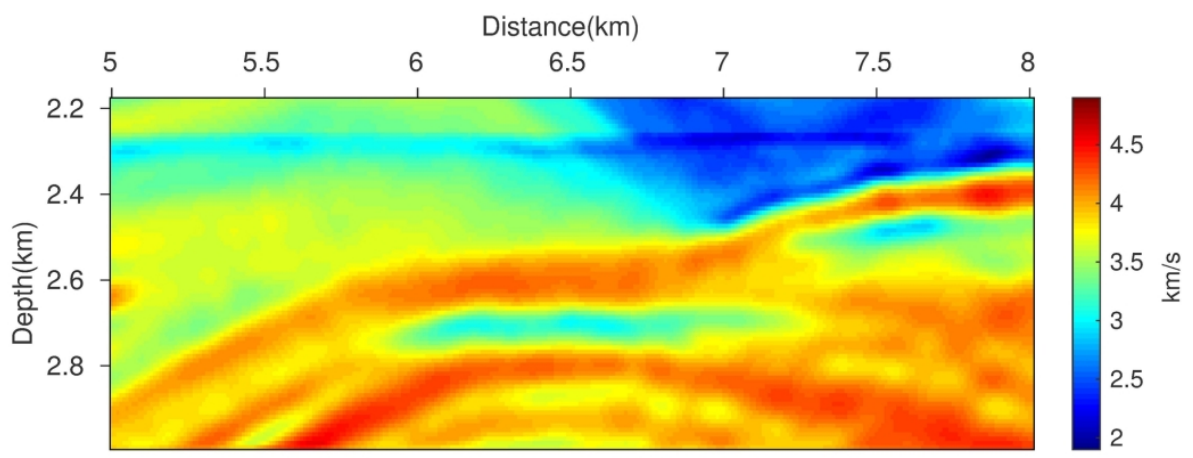

Figure 10. (c) The inverted baseline model corresponding to the noisy data shown in Figure 10(a). $188 \times 71 \mathrm{~mm}(300 \times 300 \mathrm{DPI})$ 


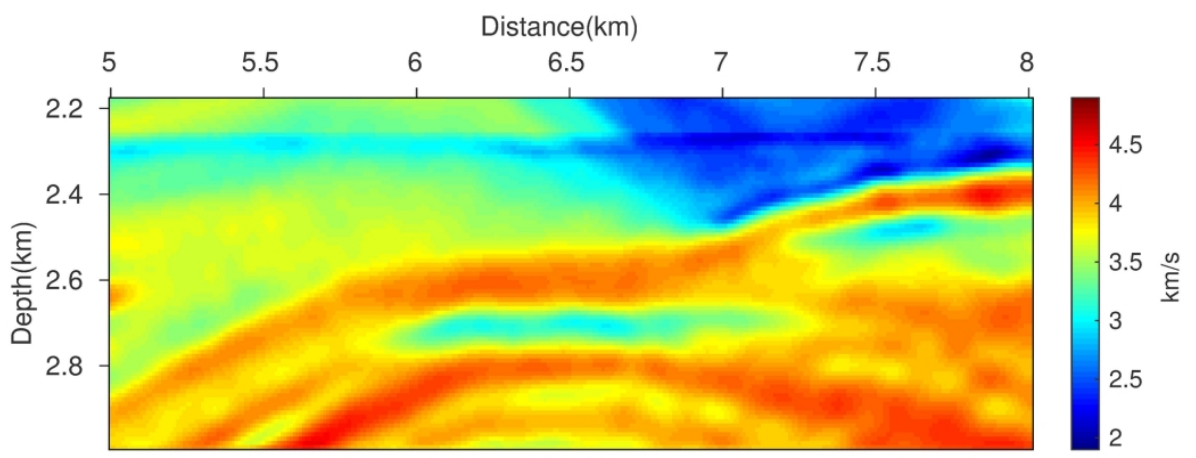

Figure 10. (d) The inverted baseline model corresponding to the noisy data shown in Figure 10(b). $188 \times 71 \mathrm{~mm}(300 \times 300 \mathrm{DPI})$ 


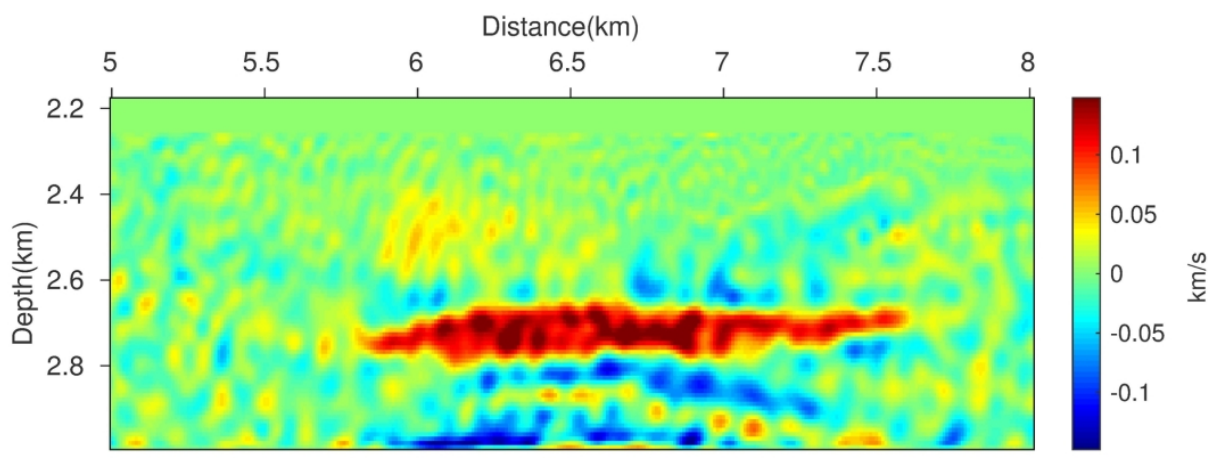

Figure 10. (e) the recovered time-lapse changes corresponding to the noisy data shown in Figure 10(a). $188 \times 71 \mathrm{~mm}(300 \times 300 \mathrm{DPI})$ 


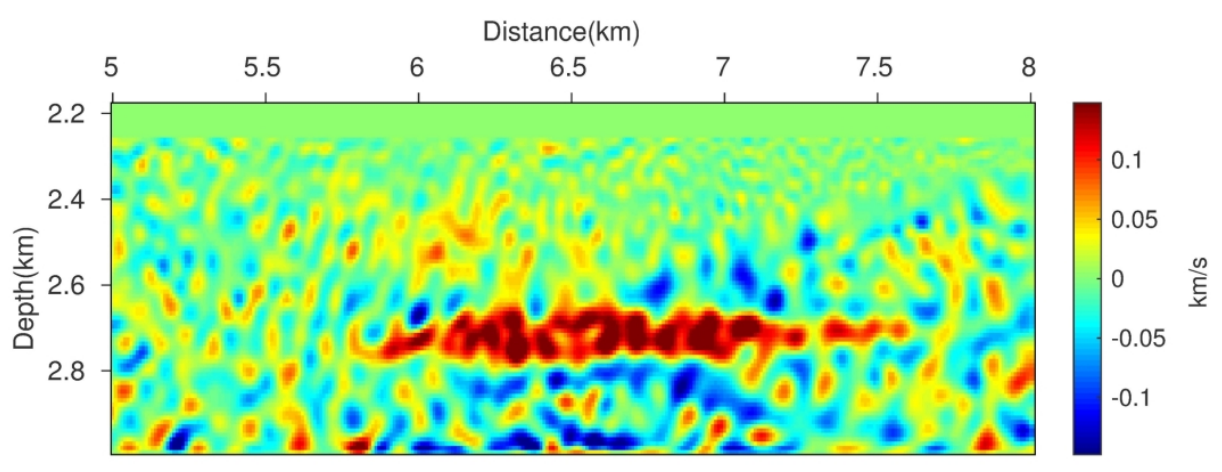

Figure 10. (f) the recovered time-lapse changes corresponding to the noisy data shown in Figure $10(\mathrm{~b})$. $188 \times 71 \mathrm{~mm}(300 \times 300 \mathrm{DPI})$ 


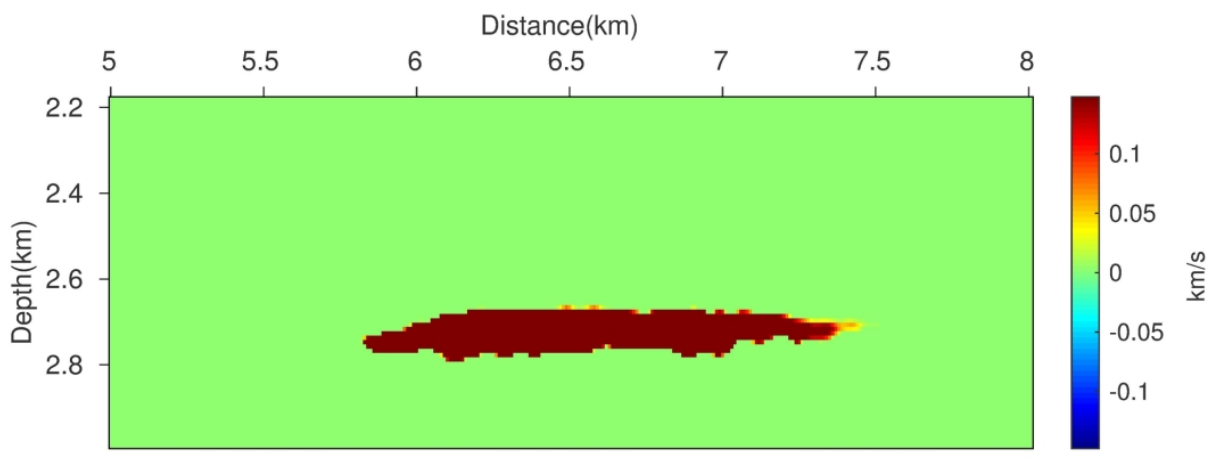

Figure 10. (g) the predicted prior model in the target zone corresponding to the noisy data shown in Figure 10(a).

$188 \times 71 \mathrm{~mm}(300 \times 300$ DPI $)$ 


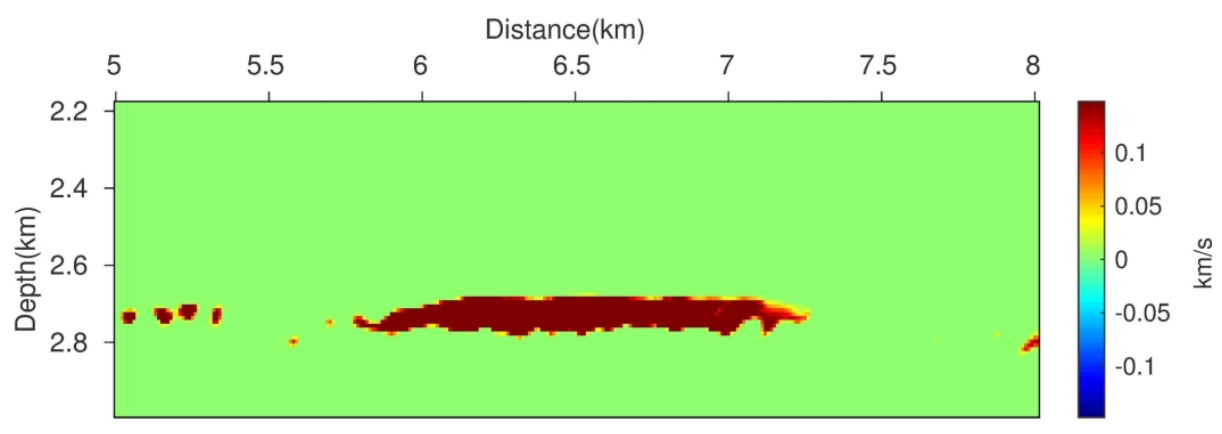

Figure 10. $(\mathrm{h})$ the predicted prior model in the target zone corresponding to the noisy data shown in Figure $10(b)$.

$185 \times 63 \mathrm{~mm}(300 \times 300 \mathrm{DPI})$ 


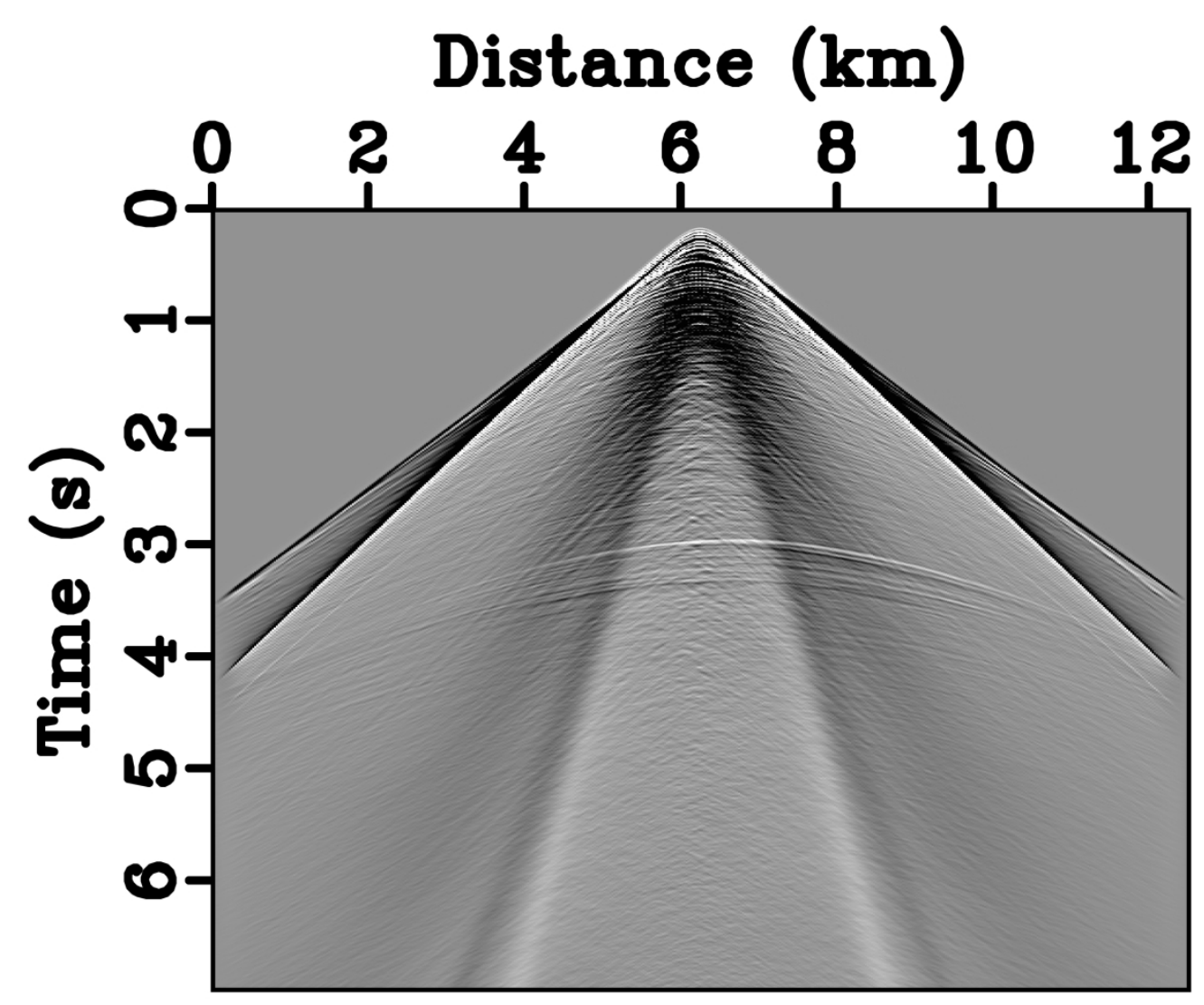

Figure 11. (a) A shot gather of the pressure component for the baseline model $286 \times 239 \mathrm{~mm}(300 \times 300 \mathrm{DPI})$ 


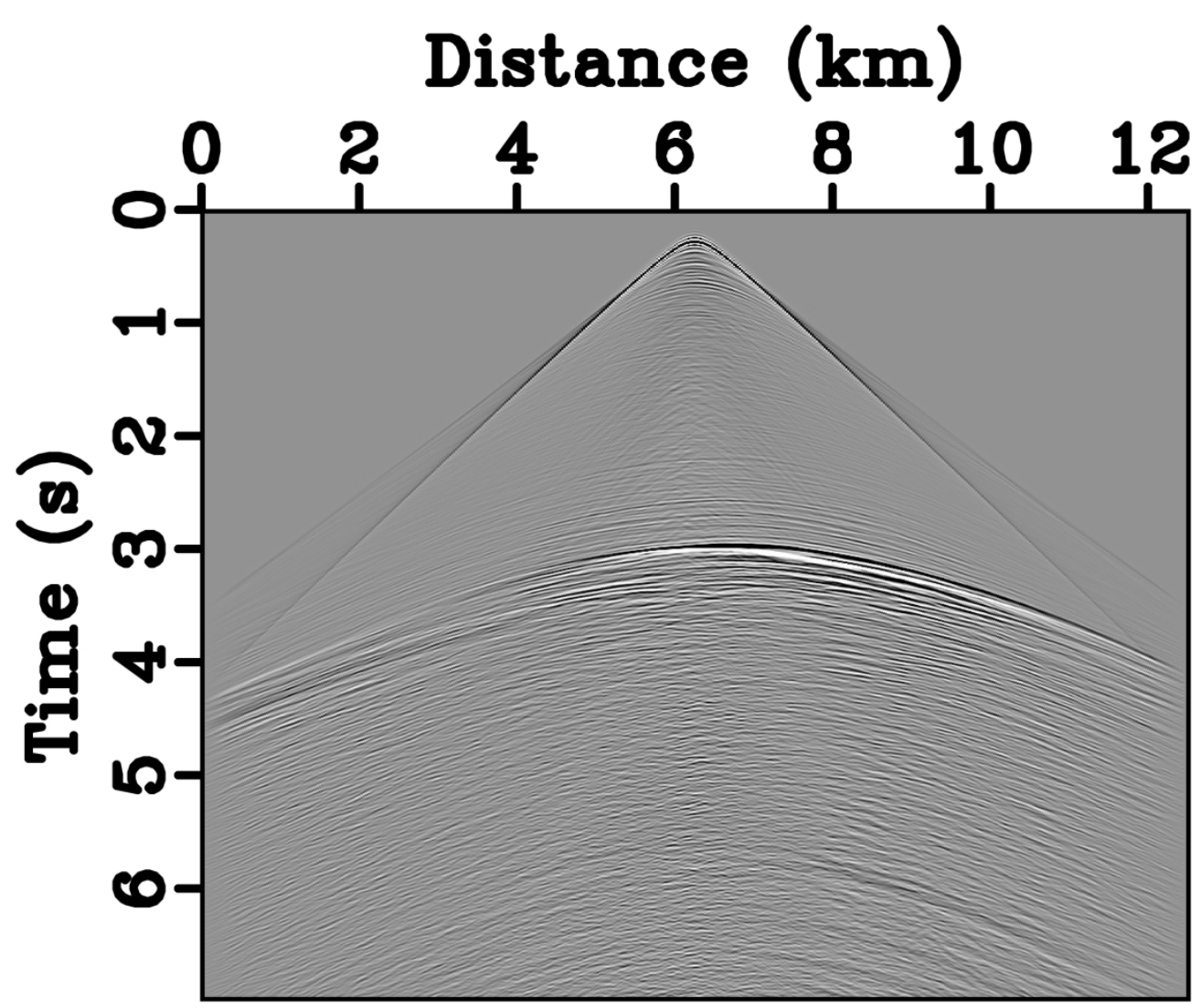

Figure 11. (b) the corresponding time-lapse data difference for the SEAM example. $286 \times 239 \mathrm{~mm}(300 \times 300 \mathrm{DPI})$ 


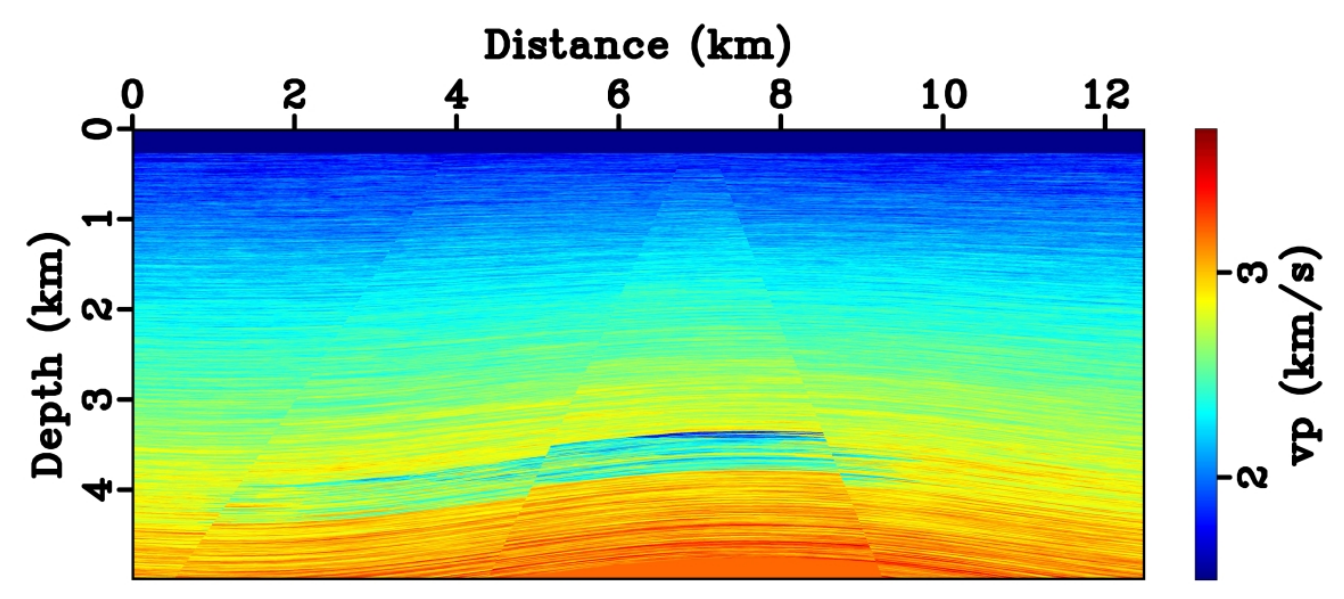

Figure 12. The baseline SEAM model: (a) P-wave velocity $549 \times 239 \mathrm{~mm}(300 \times 300$ DPI $)$ 


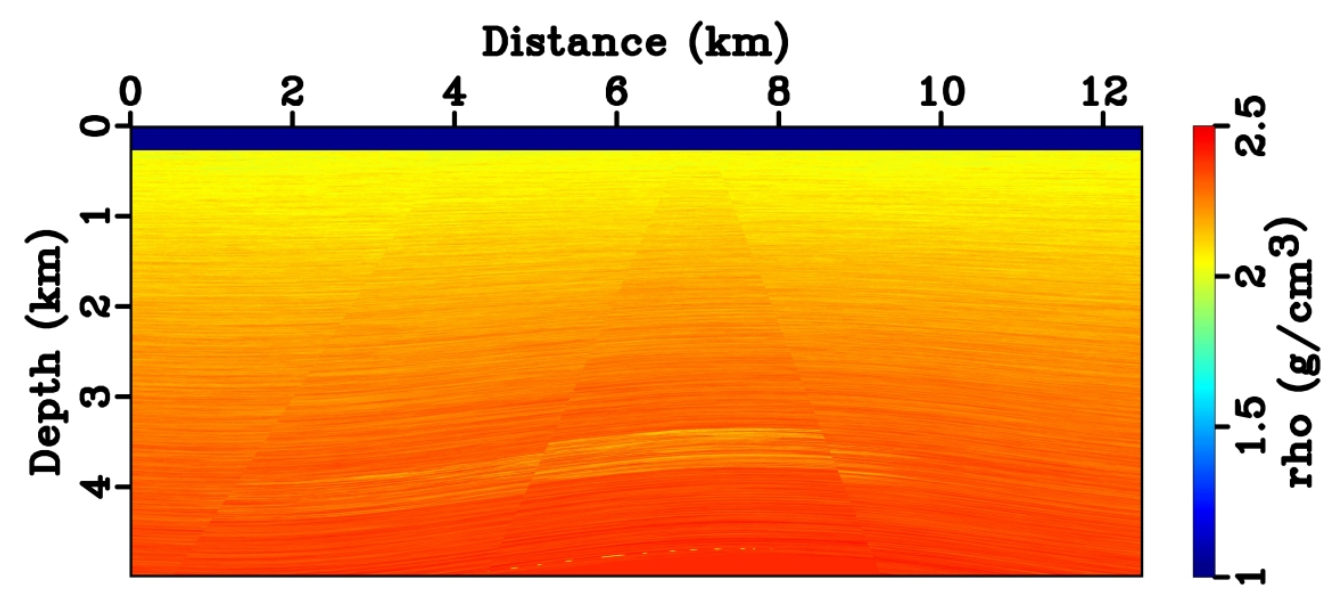

Figure 12. The baseline SEAM model: (b) density. $549 \times 241 \mathrm{~mm}(300 \times 300$ DPI $)$ 


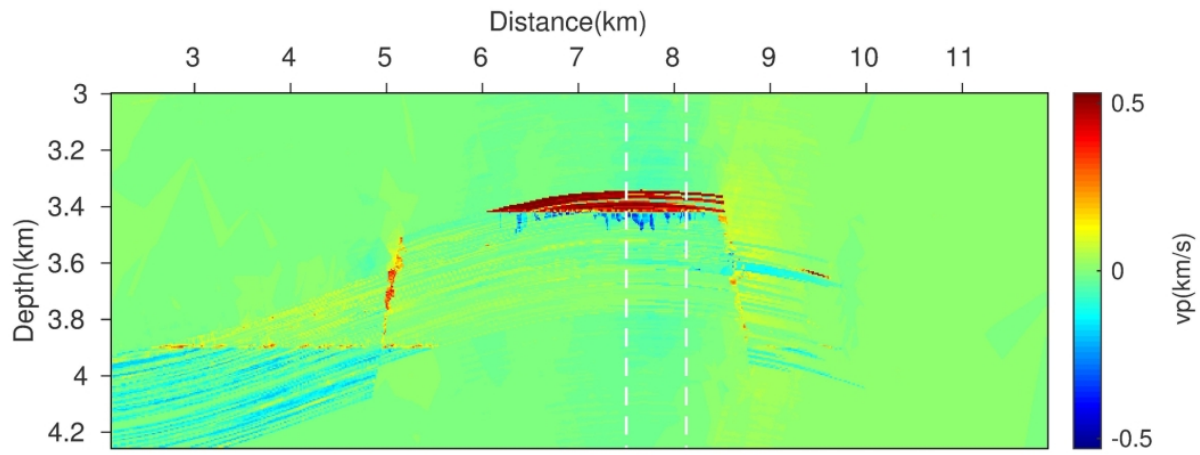

Figure 12. (c) The true time-lapse velocity changes in the target zone for the SEAM example. $188 \times 71 \mathrm{~mm}(300 \times 300 \mathrm{DPI})$ 


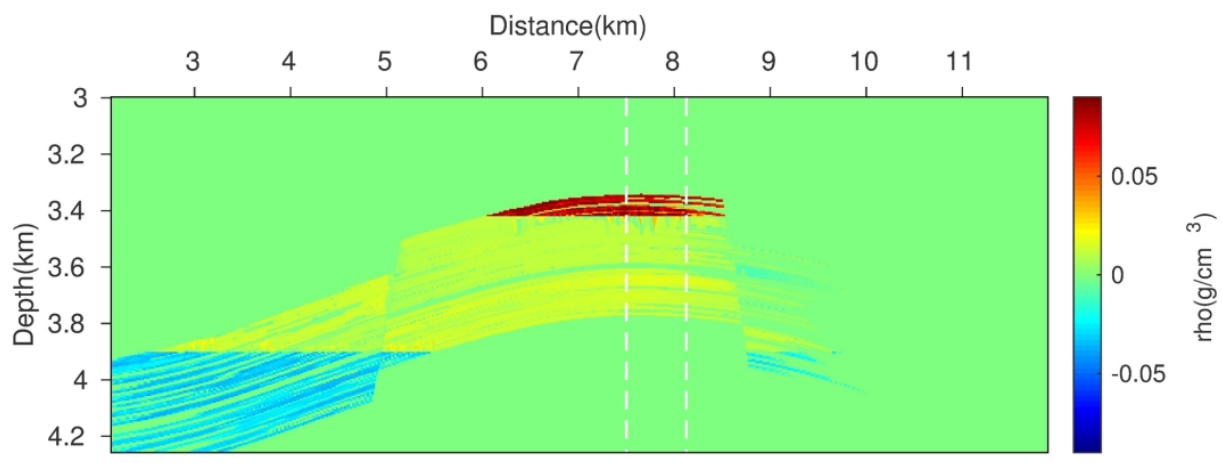

Figure 12. (d) The true time-lapse density changes in the target zone for the SEAM example. $188 \times 71 \mathrm{~mm}(300 \times 300$ DPI $)$ 


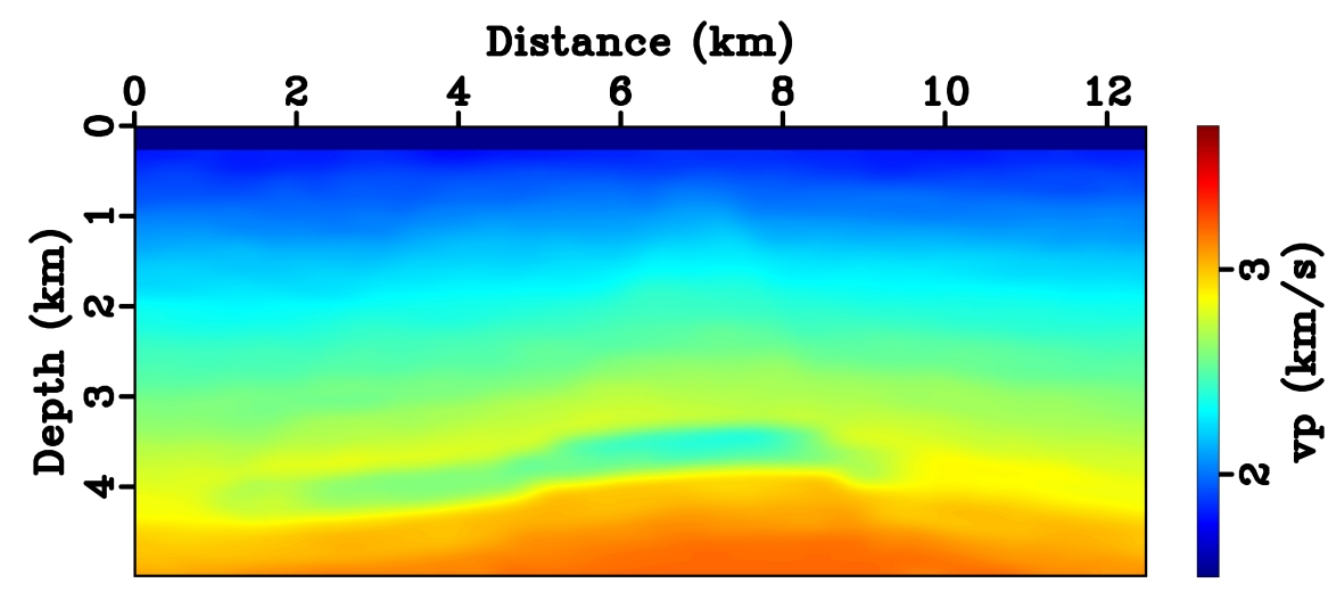

Figure 13. The starting model for the SEAM example: a smoothed version of the average of the baseline and monitor models, (a) velocity.

$549 \times 239 \mathrm{~mm}(300 \times 300$ DPI $)$ 


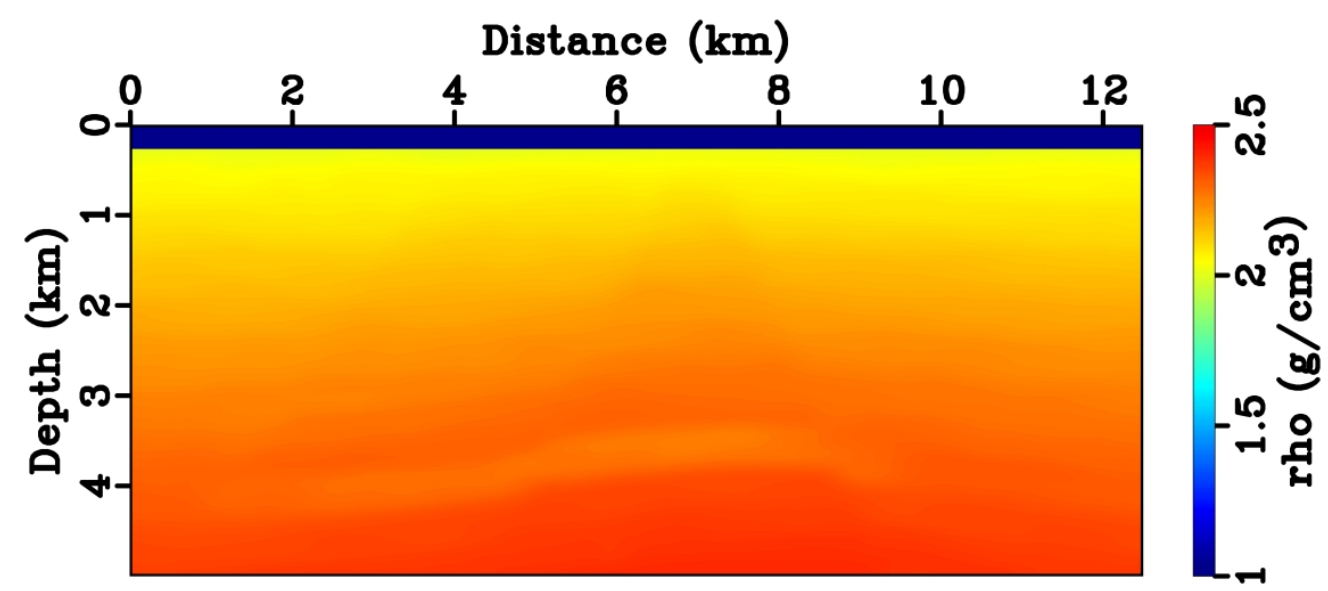

Figure 13. The starting model for the SEAM example: a smoothed version of the average of the baseline and monitor models, (b) density.

$549 \times 241 \mathrm{~mm}(300 \times 300$ DPI $)$ 


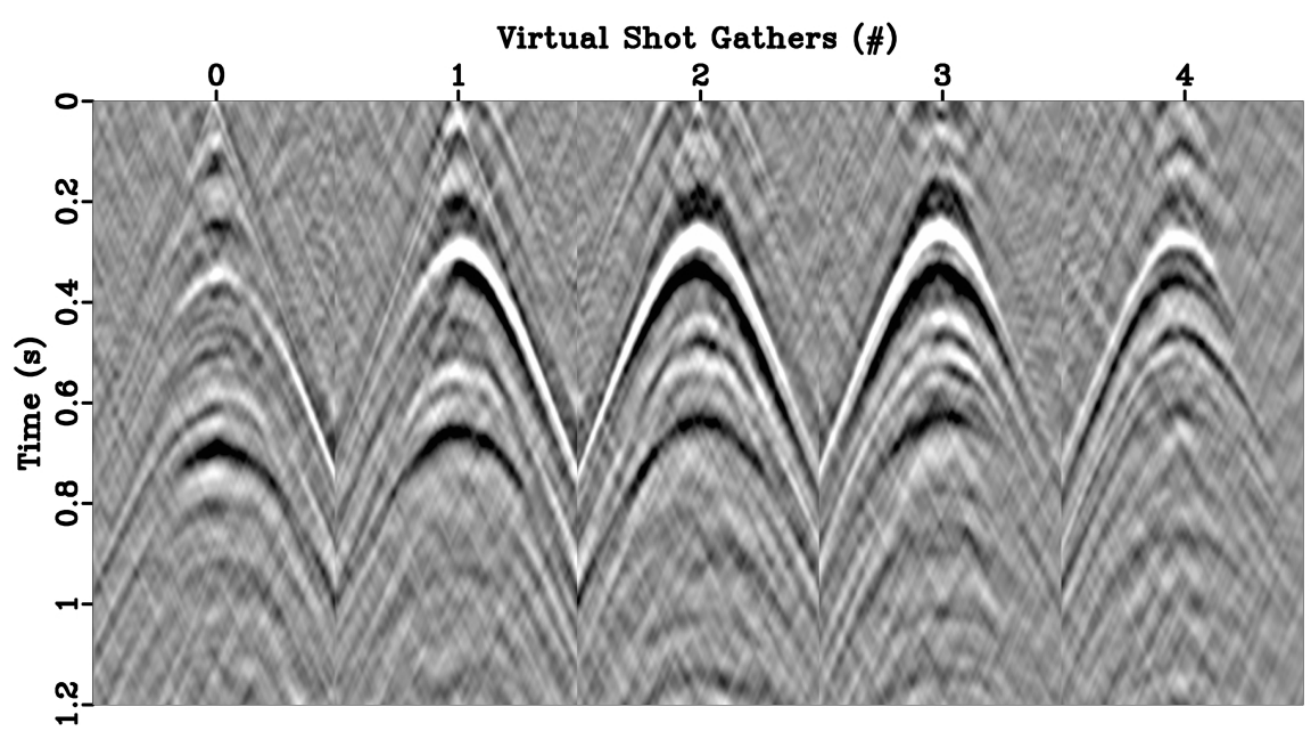

Figure 14. (a) The virtual shot gathers for baseline at the datum level for the SEAM example. $419 \times 230 \mathrm{~mm}(300 \times 300$ DPI $)$ 


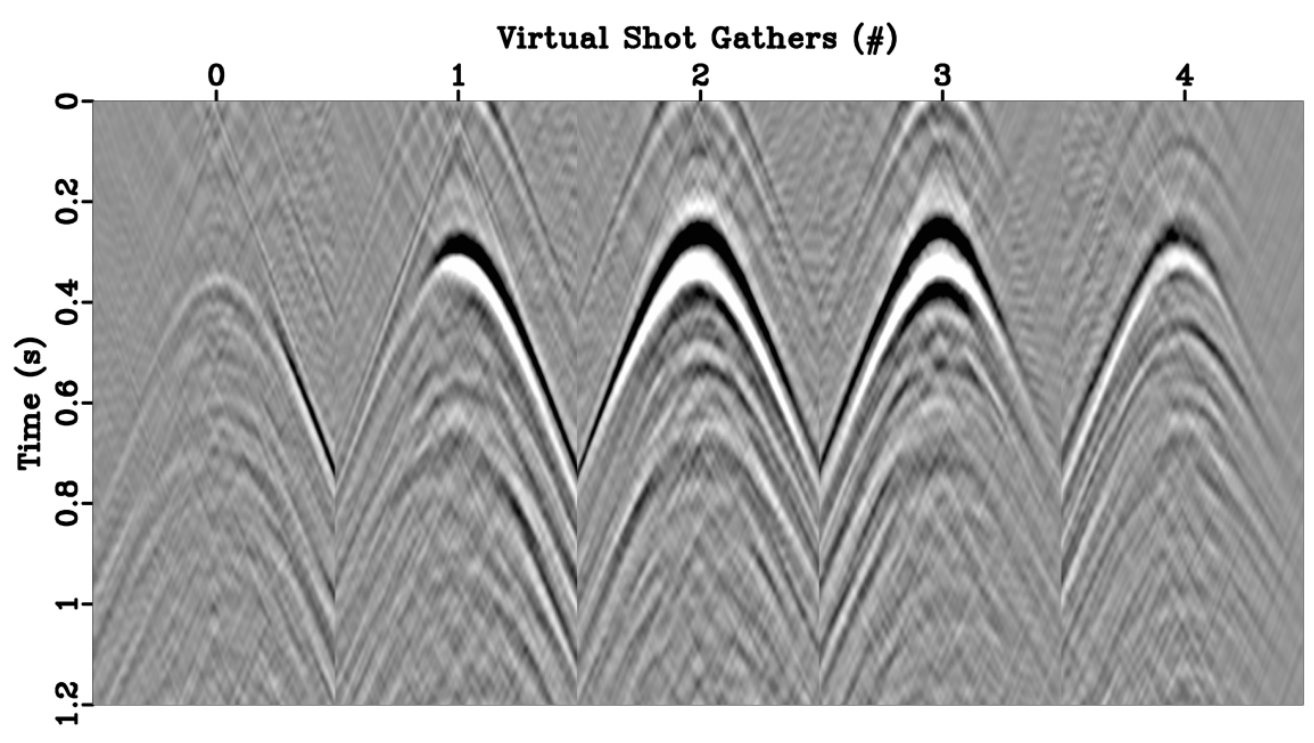

Figure 14. (b) the time-lapse data difference for the virtual survey for the SEAM example. $419 \times 230 \mathrm{~mm}(300 \times 300 \mathrm{DPI})$ 


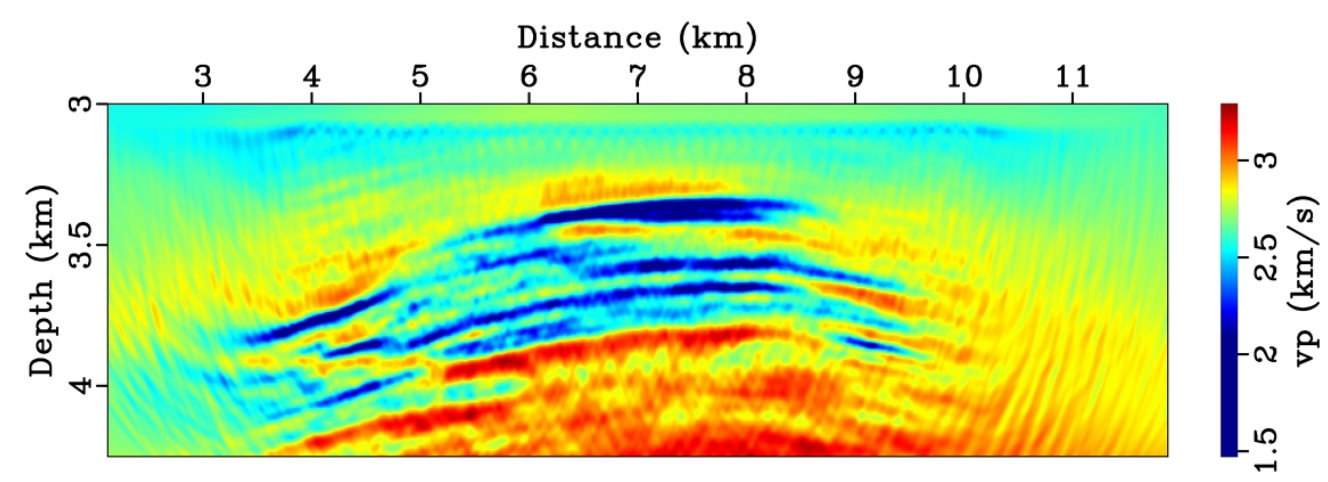

Figure 15. The SEAM example: (a) the inverted baseline model in the target zone $700 \times 246 \mathrm{~mm}(300 \times 300 \mathrm{DPI})$ 


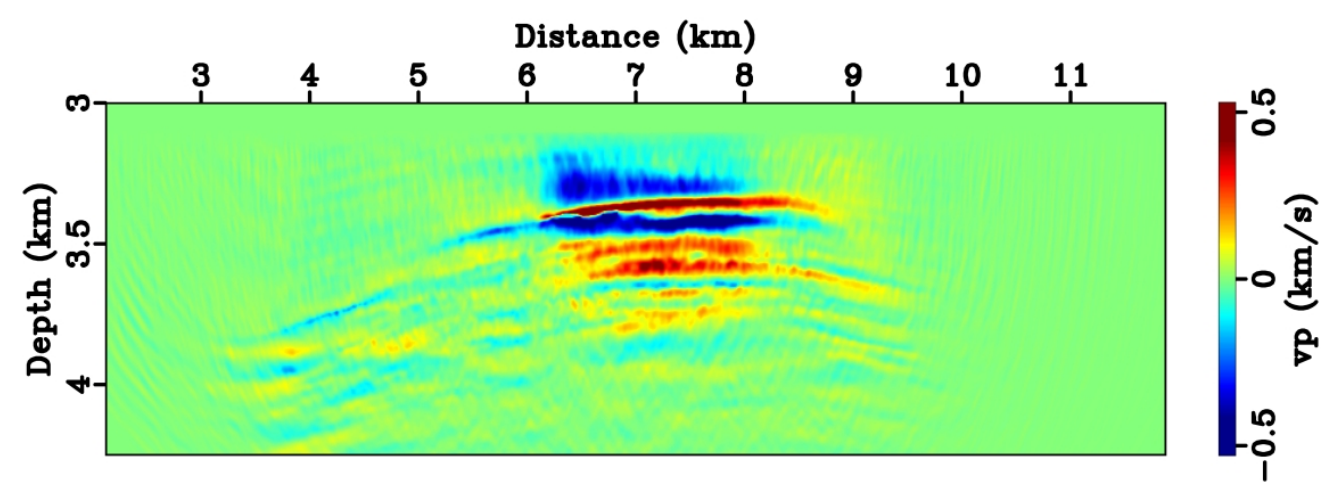

Figure 15. (b) the standard target-oriented time-lapse inversion result

$700 \times 251 \mathrm{~mm}(300 \times 300$ DPI) 


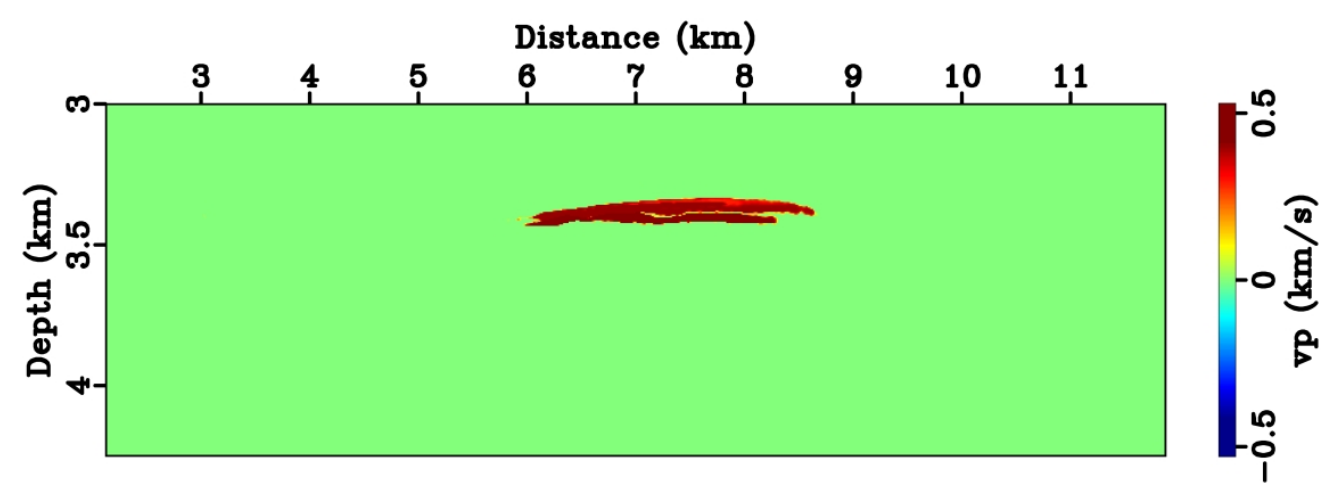

Figure 15. (c) the predicted prior model using deep learning $700 \times 251 \mathrm{~mm}(300 \times 300$ DPI $)$ 


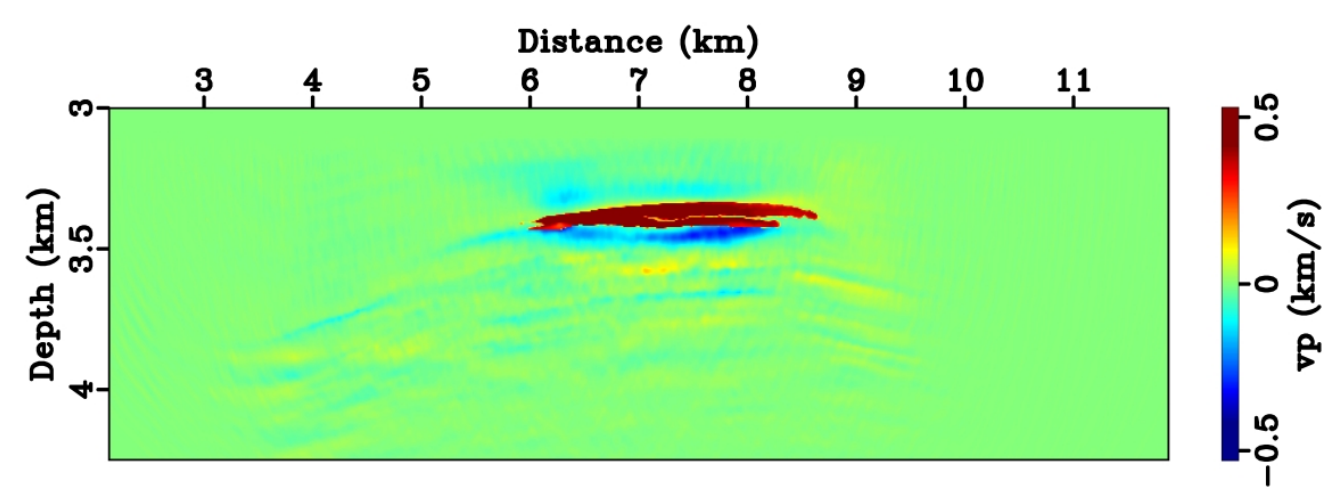

Figure 15. (d) the final inverted velocity changes regularized by the prior model in Figure $15 \mathrm{c}$.

$700 \times 251 \mathrm{~mm}(300 \times 300$ DPI $)$ 


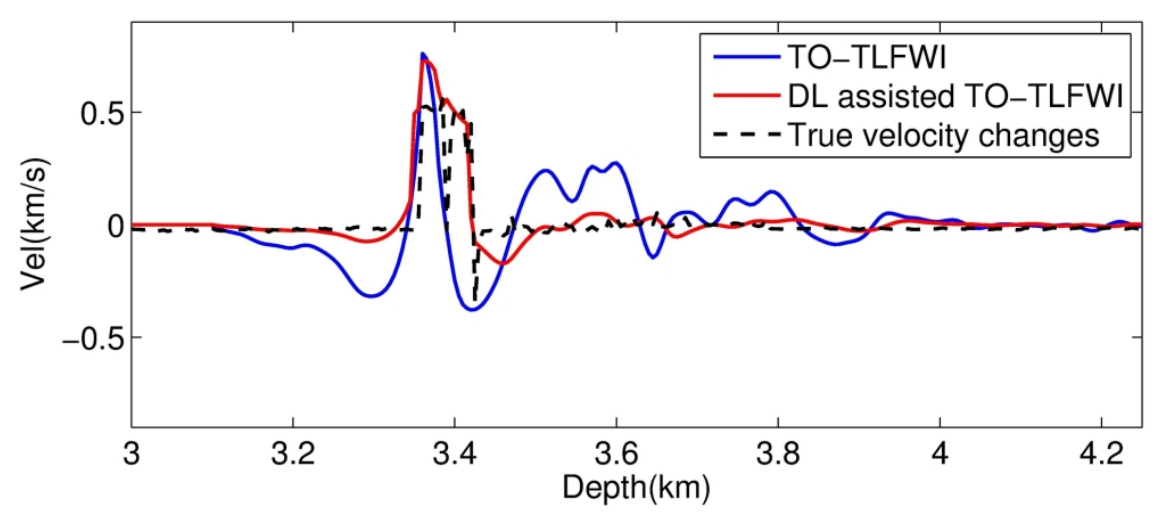

Figure 16. The vertical profiles of the inverted and true velocity changes at $x=7.0 \mathrm{~km}$ for the SEAM example.

$199 \times 80 \mathrm{~mm}(300 \times 300 \mathrm{DPI})$ 
DATA AND MATERIALS AVAILABILITY

Data associated with this research are available and can be obtained by contacting the corresponding author. 